\title{
RECURSOS Y DIETAS HUMANAS EN LAGUNA LLANCANELO (MENDOZA; NORDPATAGONIA): UNA DISCUSIÓN ISOTÓPICA DEL REGISTRO ARQUEOLÓGICO
}

\author{
MIGUEL GIARDINA", MERCEDES CORBAT", CLARA OTAOLA", LAURA SALGÁN"***, \\ ANDREW UGAN" ${ }^{\cdots+\cdots}$, GUSTAVO NEME" \& ADOLFO GIL
}

\section{RESUMEN}

Este trabajo intenta caracterizar la dieta humana mediante los isótopos estables $\left(\delta^{13} \mathrm{C}\right.$ y $\delta^{15} \mathrm{~N}$ en colágeno y $\delta^{13} \mathrm{C}$ y $\delta^{18} \mathrm{O}$ en hidroxiapatita). Los resultados se discuten en primer lugar con la ecología isotópica generada para esta zona. Luego estas tendencias son comparadas con las generadas por el modelo de mezcla isotópica aplicando el software SISUS y, finalmente, comparando esto con el registro zooarqueológico de Llancanelo. En esta escala los resultados muestran una significativa variabilidad tanto en isótopos humanos como en el registro zooarqueológico que podrían segregarse en las tendencias del sector Norte y sector Oeste de la laguna. Aún así, hay similares tendencias en el humedal: isótopos y fauna señalarían una baja importancia de guanaco y reidos, y una mediana importancia de recursos acuáticos y animales terrestres pequeños. A pesar de no haber aún registro que lo confirme, los isótopos simulados por SISUS proponen una fuerte explotación de algarrobos (Prosopis ssp.) y en algunos casos también molle (Schinus polygamus).

La información isotópica del conjunto humano arqueológico procedente de Llancanelo no muestra diferencias significativas con el resto de las muestras humanas del sur de Mendoza, pero, analizadas por sectores de la laguna, las del Oeste tienen mayor similitud con las dietas isotópicas de Payunia/Planicies Orientales y las del Norte con las de Piedemonte. Sobre esta base se propone a Llancanelo como un espacio de convergencia poblacional que fue explotado complementariamente con otras áreas del sur de Mendoza.

PALABRAS CLAVE: dieta humana, isótopos estables, humedal, Nordpatagonia, Argentina.

* Museo de Historia Natural de San Rafael - CONICET IANIGLA. Parque Mariano Moreno s/n, 5600, San Rafael Mendoza. Argentina. miguelgiardina@arqueologiamendoza.org, claraotaola@arqueologiamendoza.org, gustavoneme@ arqueologiamendoza.org.

" CONICET - Asociación de Investigaciones Antropológicas. Bartolomé Mitre 1131 - $7^{\circ}$ piso - depto. "G" 1036 Buenos Aires. mercedescorbat@yahoo.com.ar.

Museo de Historia Natural de San Rafael - IANIGLA/ ICES Malargüe. Avenida Esquivel Aldao y J. Rodriguez s/n, 5613, Malargüe, Mendoza. Argentina. mlaurasalgan@gmail.com.

Far Western Anthropological Research Group inc.; Department of Anthropology, University of Utah y Museo de Historia Natural de San Rafael- IANIGLIA Parque Mariano Moreno s/n, 5600, San Rafael Mendoza. Argentina. andrew.ugan@gmail. com.

Museo de Historia Natural de San Rafael - CONICET/IANIGLA y Facultad de Filosofía y Letras, Universidad Nacional de Cuyo. Parque Mariano Moreno s/n, 5600, San Rafael Mendoza. Argentina. agil@mendoza-conicet.gob.ar. 


\title{
RESOURCES AND HUMAN DIET IN LLANCANELO (MENDOZA; NORDPATAGONIA): ONE DISCUSSION ARCHAEOLOGICAL RECORD
}

\begin{abstract}
This study uses stable isotope data to improve our understanding of human exploitation of Llancanelo lagoon, a saline lake and wetland located between the Monte and Patagonian deserts of Mendoza, Argentina. We collect stable $\mathrm{C}, \mathrm{N}$, and $\mathrm{O}$ isotope data from human remains from the eastern and northern margins of Llancanelo and compare them with $\mathrm{C}$ and $\mathrm{N}$ data from an array of local plants and animals, zooarchaeological data from local archaeological sites, and C, N, and O data from human remains elsewhere in southern Mendoza. The isotopes and archaeofaunas from Llancanelo show a low reliance on guanaco and rheids and a moderate reliance on aquatic resources and small, terrestrial game. Although there are no corresponding archaeological remains, the isotope data also suggest a heavy use of mesquite (Prosopis ssp.) and Hardee peppertree (Schinus polygamus). Carbon and nitrogen isotope data from the human remains reveal no significant differences between samples from the Llancanelo area and human remains from the rest of Mendoza. When analized by subarea, however, humans from the west of the lake are more similar to individuals from La Payunia and the eastern plains and humans from the south of Llancanelo more similar to individuals from the Andean piedmont. Based on this information we suggest that Llancanelo acted as a point of population convergence and was exploited in a fashion complementary to other areas in the south of Mendoza.
\end{abstract}

KEY WORDS: human diet, stable isotope, wetland, Nordpatagonia, Argentina.

\section{INTRODUCCIÓN}

El centro occidente de Argentina, específicamente el sur de Mendoza, es un área de alta variabilidad ambiental. Por ello se espera una diversidad de situaciones conductuales y de estrategias humanas que podrían estar integradas espacial y temporalmente en distintas escalas. Es así que necesitamos profundizar el rol de diversos sectores en las estrategias humanas. Laguna Llancanelo es ecológicamente contrastante con el resto del sur de Mendoza. La investigación arqueológica de la misma se orienta al rol que para los humanos tendrían estos humedales en contextos áridos. Este trabajo específicamente explora la dieta humana de individuos recuperados en este humedal y la compara con otras muestras del sur de Mendoza con el objeto de discutir la homogeneidad/heterogeneidad de sus dietas, considerar su grado de variación y evaluar los rangos de acción en la macro escala para entender los procesos involucrados en la relación humanoambiente. Para ello, por un lado se presentan datos de la ecología isotópica de Llancanelo, mientras que por el otro $-y$ atendiendo a esa información de base-, se interpretan los resultados de isótopos estables $\left({ }^{13} \mathrm{C},{ }^{15} \mathrm{~N}\right)$ obtenidos en las muestras óseas humanas de la laguna. Asimismo se interpretan estos resultados junto a datos de ${ }^{18} \mathrm{O}$ que son proxy de movilidad y residencia individual. Este trabajo explora el potencial de los modelos de mezcla isotópica para estimar las fuentes probables que explican la dieta isotópica humana de Llancanelo. Las tendencias obtenidas se discuten con el registro arqueológico del área y las de isótopos estables conocidas para otras muestras humanas del sur de Mendoza.

Estudios previos señalan una importante variabilidad en las dietas humanas del sur de Mendoza (Gil et al. 2010). Para comprender dichos patrones se requiere ajustar a una escala espacial y temporal más pequeña las variaciones de los individuos analizados en comparación con la escala espacial mayor antes mencionada. Los humedales, como Llancanelo, son generalmente entendidos como áreas marginales para el uso humano (Nicholas 1998). Desde el momento en que los humedales formaron parte del paisaje mayor ocupado por los humanos es necesario incluirlos para lograr una visión arqueológica representativa de las relaciones entre las poblaciones y su entorno en el pasado (Nicholas 1998). Como ha señalado 
Binford (ver Nicholas 1998:720) wetlands always appear as excepcional case; es oportuno discutir si esa es la situación de Llancanelo y en ese caso, qué implicaciones tiene en el proceso regional.

\section{Ocupación Humana y Estructura Ambiental de Llancanelo}

Llancanelo se emplaza en la intersección del pedemonte surmendocino con La Payunia (Fig. 1). La laguna, específicamente, es un cuerpo de agua salina situado a unos $1300 \mathrm{msnm}$ en un ambiente semidesértico. Actualmente el espejo de agua se encuentra reducido a casi una tercera parte debido a la pérdida del equilibrio hídrico natural que existía entre el ingreso de agua superficial (río Malargüe y arroyos tributarios), surgente y subterránea, y el egreso de agua por evapotranspiración y escurrimiento. Dado que todos los aportes de agua son principalmente de origen níveo, la laguna presenta fluctuaciones estacionales en la localización de sus márgenes y en la profundidad del espejo de agua (con una media de $30 \mathrm{~cm}$ ), un marcado crecimiento durante el verano y una reducción en el invierno. Diversos estudios señalan variaciones en su tamaño, posiblemente como consecuencia de cambios climáticos regionales (Isla et al. 2005) pero aún se conoce muy poco de la magnitud y cronología de estos procesos en el largo plazo. Biogeográficamente, la laguna pertenece a un área transicional entre los desiertos del Monte y Patagónico, recibiendo elementos vegetales pampeanos, andinos y sub-andinos. En este sistema endorreico con depósitos salinos y ambientes de manglar, se distinguen diferentes hábitats de características propias: esteros y estuarios, llanura salina anegadiza, médanos, espejo de agua, costas y playas.

$\mathrm{Si}$ bien existen hallazgos arqueológicos, que conforman las colecciones de los museos regionales de Malargüe y San Rafael desde hace varias décadas, los estudios sistemáticos se iniciaron aproximadamente en los años 80 aunque tomando mayor intensidad en este siglo (Gil \& Neme 2006; Corbat et al. 2009; Giardina et al. 2012). La evidencia señalaba el uso de esta laguna desde hace unos 2.000 años pero recientes estudios (Giardina et al. 2012) profundizaron esta tendencia temporal hacia los inicios del Holoceno

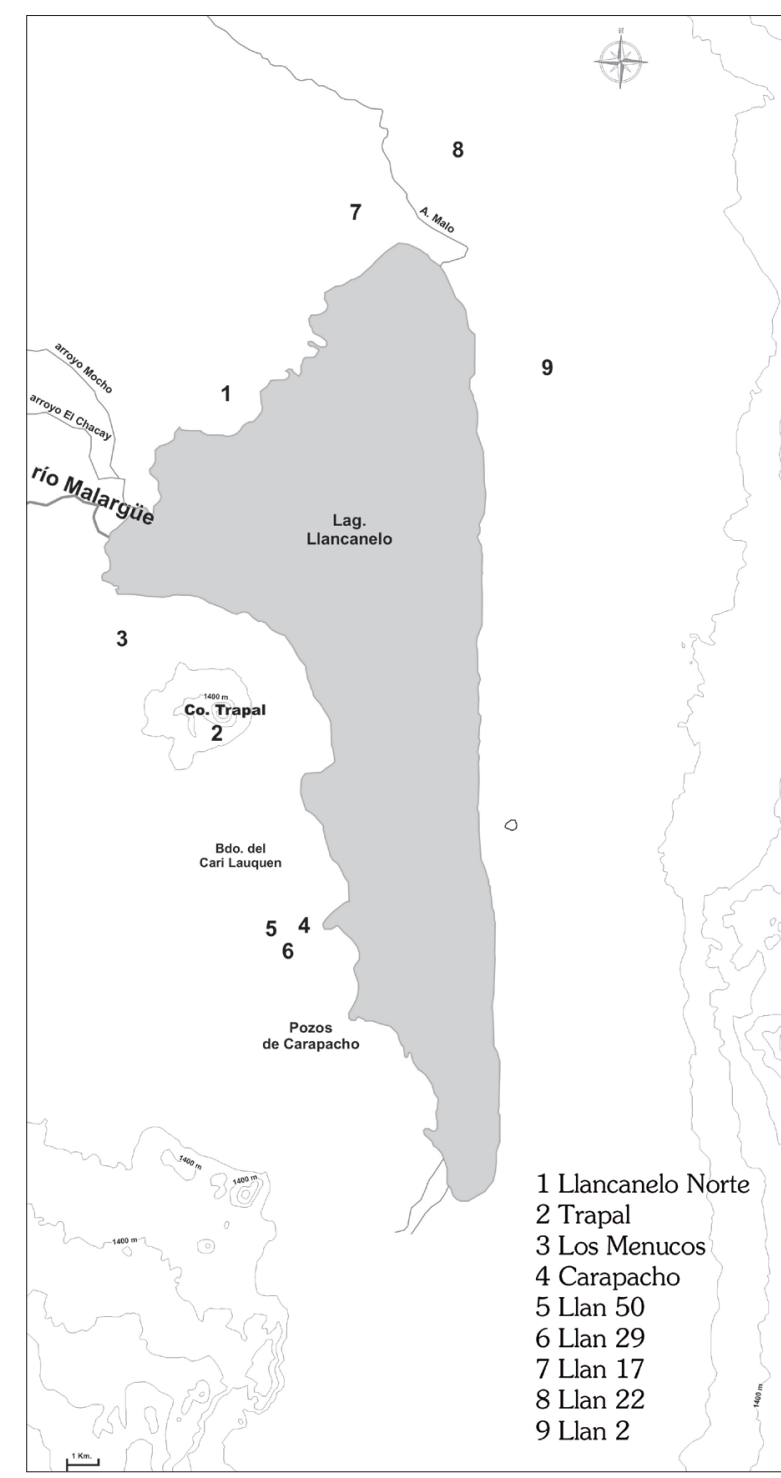

Fig. 1. Sitios y sectores de Llancanelo mencionados en el texto.

tardío (ver más adelante). Los datos con que se cuenta provienen principalmente de la colección bioantropológica del Museo Regional Malargüe (Gil et al. 2007) y de trabajos de campo que generaron patrones de distribuciones superficiales y materiales en estratigrafía (Gil \& Neme 2006; Bonnat \& Franchetti 2010). Las distribuciones superficiales señalaron una mayor densidad de materiales en el sector Norte que en el Este de la laguna, careciendo de datos sistemáticos para el Oeste y Sur. Los recientes trabajos estratigráficos muestran una mayor tasa de depositación de ítems 
arqueológicos, mayor diversidad de elementos y mayor profundidad temporal en el sector Oeste en relación a los sectores Este y Norte (Gil y Neme 2006).

\section{ISÓTOPOS ESTABLES: ASPECTOS METODOLÓGICOS GENERALES}

Los isótopos estables han mostrado sobradamente su solidez para mejorar nuestro conocimiento de los procesos ecológicos y arqueológicos principalmente enfocando a la reconstrucción de cadenas tróficas y las dietas humanas (Schoeninger \& De Niro 1984; Ambrose 1993; Pate 1997; Koch 1998; Fry 2008; Panarello et al. 2010; Tessone y Belardi 2010; Gil et al. 2012; Barberena 2013). Como Panarello et al. (2010) señalan, los isótopos estables se han convertido en una línea de evidencia con fuerte desarrollo, la cual ha sido aplicada a temas diversos como paleodieta, paleotemperaturas y movilidad humana, entre otros.

El método se basa en la propiedad de discriminación isotópica diferencial. Para el caso del carbono, esta discriminación $\left({ }^{13} \mathrm{C} /{ }^{12} \mathrm{C}\right)$ permite diferenciar entre vegetales con distintos mecanismos fotosintéticos $\left(\mathrm{C}^{3}, \mathrm{C}^{4}\right.$ y $\left.\mathrm{CAM}\right)$. Por ejemplo, el maíz es una planta con fotosíntesis $\mathrm{C}^{4}$, por lo que puede fácilmente discriminarse en contextos ecológicos donde los recursos tengan otro camino fotosintético. Isótopos de otros elementos, como el nitrógeno $\left({ }^{15} \mathrm{~N} /{ }^{14} \mathrm{~N}\right)$ y el oxígeno $(18 \mathrm{O} / 16 \mathrm{O})$ permiten discutir temas vinculados a la movilidad y las cadenas tróficas (Panarello et al. 2010). Para estos estudios se pueden procesar diferentes materiales (Ambrose 1993); en el presente trabajo se emplea material óseo.

En la práctica se ha preferido usar la relación entre el número de moléculas que contiene el isótopo más pesado y el más liviano (Barberena 2002; Panarello et al. 2010). Dado que los valores de esta relación son muy pequeños, los mismos se expresan en forma más sencilla basados en la desviación isotópica (ठ), la cual señala cuánto se aparta la relación isotópica de la muestra en estudio respecto a la relación que presenta en el patrón internacional ya definido:

$$
\delta=1000 \frac{R_{M}-R_{R}}{R R} \% 0
$$

donde RM es la relación isotópica en la muestra y $\mathrm{RR}$ se refiere dicho vínculo en el patrón internacional aludido. La misma situación se da para el $\delta^{15} \mathrm{~N}$ en la asociación entre ${ }^{15} \mathrm{~N} /{ }^{14} \mathrm{~N}$ y para $\delta^{18} \mathrm{O}$ en la relación 18O/16O. Los patrones internacionales con los que se refieren estos vínculos corresponden a V-PDB para el carbono, AIR para el nitrógeno y V-SMOW para el oxígeno (Panarello et al. 2010).

La distribución de los valores isotópicos del carbono en plantas $\mathrm{C}^{3}$ tiene un valor modal de $-27 \%$, comprendido en un rango de $-34 \%$ o $-22 \%$ o (Heaton 1999). Por su parte la distribución de valores de carbono para plantas con mecanismo fotosintético $C^{4}$ varía entre $-16 \%$ a a $-7 \%$ o, con valor modal de $-13 \%$ o (Pate 1997; Tessone 2010). Por ello, en el centrooeste argentino se asume que el colágeno de un individuo con una dieta basada en recursos $\mathrm{C}^{3}$ tendrá valores aproximados de $\delta^{13} \mathrm{C}$ de $-21.5 \%$ 。-PDB, con un rango entre - $17 \%$ o - $23 \%$ o V-PDB. Para aquellos con una dieta basada en recursos $\mathrm{C}^{4}$ su valor en el colágeno óseo estará en el orden de -7.5\% V-PDB, con un rango de entre $-14 \%$ o y $-7 \%$ o V-PDB. Entre esos rangos se encuentran dietas humanas que han sido consideradas mixtas (Coltrain y Leavitt 2002). Estos valores se obtienen con respecto a estándares internacionales ya establecidos (Ambrose 1993; Tykot 2006).

Según avances experimentales (Ambrose \& Norr 1993) los valores de $\delta^{13} \mathrm{C}$ basados en colágeno reflejan principalmente la fracción proteica de la dieta. Por su parte la fracción no orgánica (hidroxiapatita, o simplemente apatita) reflejaría la porción de la dieta que provee energía y se metaboliza; el $\delta^{13} \mathrm{C}$ de hidroxiapatita está en equilibrio isotópico con el bicarbonato en sangre (también con el $\mathrm{CO}^{2}$ disuelto en ella) que refleja los macronutrientes que están siendo metabolizados. Otra consideración radica en la resolución temporal según el tejido analizado. Para el caso del hueso se estima que el valor obtenido es un promedio de lo ingerido en los últimos 7-10 años, pero recientemente se ha propuesto que este periodo puede ser aún más extenso (Hedges et al. 2007). Los valores obtenidos en el material óseo muestran un fraccionamiento isotópico en ${ }^{13} \mathrm{C}$ respecto a la dieta, denominado como la diferencia entre el sustrato y la 
base (Barberena 2002), y que en el caso del colágeno se estima en un enriquecimiento estimado de 5\%, aunque existen diferencias sobre este valor según las especies y los tipos de dieta (Tessone 2010). Para la fracción inorgánica (hidroxiapatita) este fraccionamiento aún está en discusión pero se adopta el valor generalmente estipulado de un 9\%o, aunque con variaciones hasta valores cercanos al $12 \%$ o (Ambrose y Norr 1993; Tykot et al. 2009).

Los análisis de isótopos estables en nitrógeno generalmente han sido utilizados para discriminar diferencias en los niveles tróficos entre individuos (Hedges \& Reynard 2007), pero varios investigadores postulan que los valores de dicho isótopo reflejarían distintas variables ecológicas, climáticas, dietarias, nutricionales y metabólicas (Ambrose \& DeNiro 1986; Sealy et al. 1987; Hedges \& Reynard 2007; Petzke et al. 2010). A pesar de ello la relación entre el incremento en los valores de $\delta^{15} \mathrm{~N}$ asociados directamente a los niveles tróficos está bien fundada y parece no cuestionada (Hedges \& Reynard 2007).

Otro de los isótopos estables de reciente incorporación en la arqueología, particularmente de Argentina, es el $\delta^{18} \mathrm{O}$. En arqueología se utilizan los isótopos de oxígeno $\left({ }^{18} \mathrm{O} /{ }^{16} \mathrm{O}\right)$ para discutir temas de movilidad y patrones de residencia (Sponheimer \& Lee-Thorp 1999; Dupras \& Schwarcz 2001; Knudson 2009; Turner et al. 2009; Sanhueza \& Falabella 2010; Buzon et al. 2011). Estos isótopos se registran en el fosfato y carbonato de la hidroxiapatita y reflejan principalmente la composición isotópica del agua corporal, determinada básicamente por el agua ingerida en el momento de formación de los huesos y dientes (Longinelli \& Nuti 1973; Longinelli 1984; Luz et al. 1984; Sponheimer \& Lee-Thorp 1999; White et al. 2004; Turner et al. 2009). A su vez, el agua bebida varía de modo regular en relación a la latitud, elevación, patrones de lluvia y otros factores ambientales (Dansgaard 1964; Gat 1996; White et al. 1998, 2004). Por ello, si hay variaciones en los isótopos de oxígeno del agua de una región, entonces hay un potencial para explorar aspectos de la movilidad y patrones de residencia humanos (White et al. 2000, 2002, 2004; Dupras y Schwarcz 2001; Turner et al. 2009). En general, para entender los valores de humanos tanto de ${ }^{13} \mathrm{C}$ como de ${ }^{15} \mathrm{~N}$ y ${ }^{18} \mathrm{O}$, se ajustan los resultados con valores para fauna, vegetales y agua de la misma región, para generar aspectos de una ecología isotópica (Martínez del Río et al. 2009).

\section{Consideraciones Técnicas}

En este trabajo se analizaron los resultados de los isótopos estables de $\delta^{13} \mathrm{C}$ y $\delta^{15} \mathrm{~N}$ basados en el procesamiento de la fracción orgánica (colágeno) y los valores $\delta^{13} \mathrm{C}$ y $\delta^{18} \mathrm{O}$ obtenidos de la fracción inorgánica (hidroxiapatita o apatita) de especimenes óseos de faunas actuales y arqueológicas y muestras humanas arqueológicas del área de Llancanelo. Los valores $\delta^{13} \mathrm{C}$ y $\delta^{18} \mathrm{O}$ se calcularon utilizando el estándar V-PDB. El colágeno y la hidroxiapatita se obtuvieron mediante procesos desarrollados en el Laboratorio de Paleoecología Humana del Museo de Historia Natural de San Rafael siguiendo el protocolo descrito en Coltrain y Leavitt (2002). Las mediciones de los isótopos estables de $\delta^{13} \mathrm{C}, \delta^{15} \mathrm{~N}$ y $\delta^{18} \mathrm{O}$ se efectuaron en SIRFER de la University of Utah (código MSR-A), University of South Florida (código USF) y la University of Wyoming Isotopes Stable Facility (código MSR).

Para entender las tendencias en las dietas humanas inferidas desde los isótopos se requiere un modelo sobre las cadenas tróficas que contemple la variabilidad isotópica de los recursos de base (Szpak et al. 2013). Por ello, para ajustar la ecología isotópica se procesaron 68 muestras óseas de fauna actuales que incluyeron aves $(n=38)$, mamíferos $(n=19)$, reptiles $(n=1)$ y peces $(n=10)$ (Tabla 1$)$. También se incorporó información previa de camélidos y recursos vegetales (Prosopis ssp.) de sectores del sur mendocino aledaños a Llancanelo (Gil et al. 2010). Los recursos analizados incluyen tanto a grupos cuya explotación humana está demostrada (o al menos hay registro arqueológico de ellas) como otros que no. En ese sentido la ecología isotópica es un modelo abierto que no sólo apunta a discutir el consumo directo sino también los roles de estos taxones en el sistema, incluyendo allí a los humanos. Para los humanos se generó información sobre 11 muestras óseas arqueológicas de Llancanelo (Tabla 2) halladas en el sector Norte y Oeste de la laguna (Fig. 1; Tabla 2; detalles en Gil et al. 2007) con cronologías entre 2.500 y 500 años ${ }^{14} \mathrm{C}$ AP (Gil et al. 2007).

Para evaluar la integridad de las muestras se analizó en cada una de ellas la relación C:N siguiendo los criterios de Ambrose (1990) aceptándose aquellas cuyos valores estén comprendidos entre 2,9 y 3,6. 
Para analizar las relaciones y los vínculos tróficos entre las muestras se han seguido los siguientes factores de corrección: a las muestras actuales se les sumó $2 \%$ o para controlar el efecto Suess (Friedli et al. 1986). Por su parte, en la comparación entre las cadenas tróficas se utiliza para el $\delta^{15} \mathrm{~N}$ un enriquecimiento de $3 \%$ o y entre los valores de $\delta^{13} \mathrm{C}$ se estima un fraccionamiento de $5 \%$ o que corregido por las diferencias colágeno óseo/carne queda finalmente en 1\%o (Ambrose 1993).

Además de la interpretación individual y contextual de los datos se emplean aquí dos modelos que intentan ajustar distintos aspectos de la interpretación de los valores isotópicos. Por un lado, se emplea el modelo de mezcla isotópica denominado SISUS (Erhardt 2008, ver detalle y explicitaciones abajo). Por el otro, empleamos la propuesta de Kellner y Schoeninger (2007) que compara la relación entre los valores de $\delta^{13} \mathrm{C}$ en hidroxiapatita y colágeno óseo para discutir la composición de proteínas y energía en términos de aportes de recursos tipo $\mathrm{C}^{3}, \mathrm{C}^{4}$ o marino.

\section{Modelo de Mezcla Isotópica y SISUS}

Las dietas humanas promedio de los sectores Norte y Oeste se modelaron usando modelos de mezcla generados con el software de uso libre SISUS (Stable Isotope Sourcing Using Sampling) desarrollado por Erhardt (2009). Esta propuesta es un análogo directo de ISOSOURCE (Phillips \& Gregg 2003; Phillips et al. 2005) que se desarrolló para evaluar mezclas de isótopos estables en donde el número de fuentes que aportan al patrón de isótopos excede el número de isótopos disponibles para distinguir entre ellas. SISUS analiza simultáneamente múltiples combinaciones y considera diferencias en los factores de enriquecimiento, fuentes de concentración y eficiencia en la asimilación (Erhardt, 2007, 2008, 2009). Para mayor detalle del software pueden consultarse Erhardt (2007, 2008, 2009) y Ugan y Coltrain (2012).

Para la mezcla de isótopos de los individuos se utilizaron los valores de $\delta^{15} \mathrm{~N}$ obtenidos del colágeno y de $\delta^{13} \mathrm{C}$ generados de la hidrioxiapatita aceptando a ésta como un mejor reflejo de la dieta (Ambrose \& Norr 1993; Tieszen \& Fagre 1993; Schwarcz 2000; Fogel y Tuross 2003; Howland et al. 2003; Jim et al. 2006). Los datos isotópicos de las fuentes usan los valores medios de $\delta^{15} \mathrm{~N}$ y $\delta^{13} \mathrm{C}$ ofrecidos en la Tabla 1. A los fines de viabilizar el análisis se agruparon recursos con valores similares. Así se generaron los grupos "guanaco-reidos", "roedores", "patos", "huevos de reidos", "algarrobo", "molle", "perca", "pejerrey" y "armadillos". Excluimos taxa con pocos datos o con muy poco consumo humano como, por ejemplo, serpientes, gato montés y otros carnívoros, y aves carroñeras.

Definir la contribución dietaria depende de los valores estimados de la discriminación de valores $\delta^{13} \mathrm{C}$ y $\delta^{15} \mathrm{~N}$, ninguno de los cuales se conocen con precisión (Bocherens y Drucker, 2003; Hedges y Reynard, 2007; ver nuestros supuestos arriba). Para SISUS asumimos enriquecimientos en nitrógeno de $3 \%$, de carbono (carbonatos de hueso) de 9\%o, y comparamos la contribución de plantas y animales que resulte. Por su parte los valores de carbono se mantuvieron constantes, porque los efectos de incrementar la discriminación de $\delta^{13} \mathrm{C}$ se discuten junto con los cálculos de $\delta^{15} \mathrm{~N}$. El análisis de datos sobre un rango de valores de discriminación dan una idea de cuan sensitivos son los resultados para cambiar esos parámetros.

Las plantas y animales también difieren sustancialmente en su composición de $\mathrm{C}$ y $\mathrm{N}$, por lo que se requiere considerar la concentración de la fuente (Phillips \& Koch 2002; Phillips \& Gregg 2003). Para los animales terrestres y peces usamos la relación de C y $\mathrm{N}$ determinados por Ugan y Coltrain (2011). Para estos valores, la cantidad de carbón, hidrógeno, oxígeno, nitrógeno y sulfuro en moles fue calculado para cada recurso basado en la estructura molecular de azúcares, almidones, ácidos grasos, y aminoácidos y la cantidad de cada uno contenido en 100 gr de porción comestible. Esos valores se transformaron en frecuencia relativa (porcentaje) y el correspondiente valor de $\mathrm{C}$ y $\mathrm{N}$ se usó en los datos ingresados a SISUS. Para aves acuáticas, usamos el mismo método para calcular la relación de carbono y nitrógeno, basándolos en datos sobre la composición nutricional de patos silvestres (USDA 2009). Para los huevos de reidos, usamos datos sobre la estructura de huevos en Navarro et al. (2003). Para Schinus polygamus (molle) y Prosopis ssp. (algarrobo), usamos datos sobre la porcentaje de proteínas, carbohidratos, y grasas de cada recurso.

Finalmente debemos considerar aspectos de la eficiencia digestiva pues no todo el material 
consumido es absorbido por el organismo. Esto es particularmente cierto para los vegetales, donde algunas proteínas y carbohidratos están vinculados a fracciones no digeribles como las ligninas y celulosa y donde aún fracciones digeribles pueden variar en su tasa. Aquí asumimos la fracción digerible de carbón y nitrógeno de los animales en $93 \%$ y $95 \%$, y de las plantas en $80 \%$ y $85 \%$. Los datos generados por SISUS están disponibles bajo requerimiento de los autores.

\section{RESULTADOS}

Tendencias isotópicas en la fauna

Excepto dos especímenes, la relación C:N en el conjunto analizado cae entre los valores aceptables (Tabla 1). Los mencionados especímenes que caen fuera del rango presentan valores de 3,8 (muestra MSR-A292) y 5,2 (muestra MSR-A293) y son de la misma especie, Odontesthes microlepidotus. Dado que son los únicos especímenes de esa especie disponible para caracterizar la ecología isotópica de Llancanelo se optó por utilizar provisoriamente la información de la muestra MSR-A292 y eliminar la muestra MSR-A293, pero advirtiendo su situación y señalando la necesidad de profundizar análisis de este grupo para ajustar su tendencia isotópica.

La Fig. 2 se generó sobre la base de la Tabla 1, usando un desvío estándar. Las aves incluyen anátidos $(n=2)$, Cathartes aura $(n=$ $1)$, carádridos $(n=1)$, Coscoroba coscoroba ( $n=$ 22), Eudromia elegans $(n=2)$, Phalacrocorax olivaceus $(n=1)$, Phoenicopterus chilensis $(n=6)$, reidos $(n=3)$. Entre los mamíferos se incluyeron muestras de Caviidae ( $\mathrm{n}=1)$, Conepatus chingue $(n=1)$, chinchíllidos $(n=1)$, dasipódidos $(n=3)$, Lagostomus maximus $(\mathrm{n}=8)$, Myocastor coypus $(\mathrm{n}=2)$, Lycalopex griseus $(\mathrm{n}=3)$, Oncifelis geoffroyi $(n=1)$. Los reptiles están representados por una serpiente y los peces incluyen Odontesthes microlepidotus $(n=2)$ y Percichthys sp. $(n=8)$.

La Fig. 2 muestra con el valor más alto de $\delta^{15} \mathrm{~N}\left(16,9 \%\right.$ ) y más enriquecido en $\delta^{13} \mathrm{C}(-9,3 \%$ o $)$ a los especímenes de Phalacrocorax olivaceus. Esto implica para esta especie un lugar trófico significativamente más elevado que el esperable por la explotación de los recursos de esta laguna (en general $\delta^{15} \mathrm{~N} 10 \%$ en peces, ver Fig. 2). Según Petracci et al. (2009a, 2009b) los representantes de la familia Phalacrocoracidae son buceadores oportunistas; se alimentan, en su mayoría, de peces demovimientolentoy hábitosbentónicosy, en menor proporción, de invertebrados y otros organismos acuáticos. Presenta un amplio espectro trófico, pero en Argentina se alimentaría principalmente de peces $y$, en menor medida, de algunas especies de crustáceos. Los valores obtenidos no son los esperables para una explotación frecuente de Llancanelo o sectores similares por lo que puede reflejar su status migratorio. Coscoroba coscoroba, Phoenicopterus chilensis y Charadriidae también presentan valores enriquecidos en $\delta^{13} \mathrm{C}$ (promedios estimados entre -13 a -10\%o). Fundamentalmente el primero tiene una significativa variación en este isótopo, y los tres taxones con $\delta^{15} \mathrm{~N}$ esperables para niveles tróficos que incluyen los recursos de la laguna (promedios entre $11 \%$ y $8 \%$ o), aunque significativamente distintos y superiores los de Phoenicopterus chilensis (Fig. 2). Los anátidos se emplazan en la misma situación respecto al $\delta^{15} \mathrm{~N}$ y algo más empobrecidos en su valor promedio de $\delta^{13} \mathrm{C}$. En el grupo más empobrecido en $\delta^{13} \mathrm{C}$ se incluyen Cathartes aura, Eudromia elegans y Rheidae (promedios entre $-17,5 \%$ o y $-19 \%$; Fig. 2). La primera presenta el mayor valor para $\delta^{15} \mathrm{~N}$ (11\%o), la segunda un promedio de $9 \%$ y Rheidae un valor extremadamente inferior $(6,1 \%$ o).

Los mamíferos tienen valores de $\delta^{13} \mathrm{C}$ empobrecidos (entre -14 y -21\%o) menores a los de aves con dietas acuáticas (Fig. 2). Los valores de $\delta^{15} \mathrm{~N}$ son intermedios, variando entre 6,3 y $10 \%$. Entre los que presentan este isótopo en sus valores más altos están los chinchíllidos y dasipódidos. Los tres carnívoros (Conepatus chingue, Lycalopex griseus, y Leopardus geoffroyi) presentan similares valores de $\delta^{15} \mathrm{~N}$, entre 6,2 y 8,5\%o (Fig. 2) y variaciones en el $\delta^{13} \mathrm{C}$ desde aproximadamente $-14 \%$ (Conepatus chingue), hasta $-17,1 \%$ o (Lycalopex griseus), con valores intermedios para Leopardus geoffroyi (-15,8\%o). A los chinchíllidos nombrados anteriormente se le suman en el grupo de roedores las muestras de Lagostomus maximus y los cávidos, ambos con similares valores promedios de $\delta^{13} \mathrm{C}(-16 \%)$ y $\delta^{15} \mathrm{~N}(6,3 \%$ ). En el extremo inferior del modelo 
Tabla 1. Recursos analizados y sus resultados isotópicos $\left({ }^{13} \mathrm{C}\right.$ y $\left.{ }^{15} \mathrm{~N}\right)$.

\begin{tabular}{|c|c|c|c|c|}
\hline ID & Taxa & $\delta^{13} \mathrm{Ccol}$ & $\delta^{15} \mathrm{~N}$ & C:N \\
\hline MSR-A337 & Anatidae & $-17,3$ & 8,1 & 3,2 \\
\hline MSR-A337 & Anatidae & $-17,0$ & 8,3 & 3,2 \\
\hline MSR-A181 & Cathartes aura & $-19,5$ & 11,0 & 3,2 \\
\hline MSR-A327 & Caviidae & $-17,6$ & 6,3 & 3,2 \\
\hline MSR-A335 & Charadriidae & $-12,4$ & 8,6 & 3,4 \\
\hline MSR-A326 & Chinchillidae & $-21,0$ & 10,4 & 3,3 \\
\hline MSR-A321 & Conepatus chingue & $-16,1$ & 8,1 & 3,4 \\
\hline MSR-A032 & Coscoroba coscoroba & $-17,6$ & 9,9 & 3,1 \\
\hline MSR-A034 & Coscoroba coscoroba & $-14,8$ & 9,8 & 3,2 \\
\hline MSR-A035 & Coscoroba coscoroba & $-10,2$ & 6,2 & 3,2 \\
\hline MSR-A036 & Coscoroba coscoroba & $-14,6$ & 7,8 & 3,1 \\
\hline MSR-A037 & Coscoroba coscoroba & $-20,4$ & 10,6 & 3,1 \\
\hline MSR-A038 & Coscoroba coscoroba & $-11,0$ & 6,4 & 3,2 \\
\hline MSR-A059 & Coscoroba coscoroba & $-12,7$ & 8,1 & 3,2 \\
\hline MSR-A060 & Coscoroba coscoroba & $-13,5$ & 8,1 & 3,2 \\
\hline MSR-A061 & Coscoroba coscoroba & $-20,7$ & 9,7 & 3,2 \\
\hline MSR-A276 & Coscoroba coscoroba & $-15,8$ & 10,9 & 3,2 \\
\hline MSR-A277 & Coscoroba coscoroba & $-15,6$ & 11,3 & 3,2 \\
\hline MSR-A278 & Coscoroba coscoroba & $-18,1$ & 10,9 & 3,2 \\
\hline MSR-A278 & Coscoroba coscoroba & $-16,8$ & 10,6 & 3,2 \\
\hline MSR-A279 & Coscoroba coscoroba & $-15,4$ & 7,2 & 3,2 \\
\hline MSR-A280 & Coscoroba coscoroba & $-15,4$ & 9,1 & 3,2 \\
\hline MSR-A281 & Coscoroba coscoroba & $-7,5$ & 9,3 & 3,2 \\
\hline MSR-A282 & Coscoroba coscoroba & $-15,9$ & 8,4 & 3,2 \\
\hline MSR-A283 & Coscoroba coscoroba & $-11,1$ & 8,7 & 3,2 \\
\hline MSR-A284 & Coscoroba coscoroba & $-14,3$ & 8,3 & 3,2 \\
\hline MSR-A285 & Coscoroba coscoroba & $-21,3$ & 8,8 & 3,2 \\
\hline MSR-A286 & Coscoroba coscoroba & $-13,9$ & 9,6 & 3,2 \\
\hline MSR-A287 & Coscoroba coscoroba & $-14,8$ & 8,3 & 3,2 \\
\hline MSR-A338 & Eudromia elegans & $-19,8$ & 9,2 & 3,3 \\
\hline MSR-A339 & Eudromia elegans & $-19,3$ & 8,7 & 3,3 \\
\hline MSR-A041 & Lagostomus maximus & $-18,5$ & 4,7 & 3,2 \\
\hline MSR-A045 & Lagostomus maximus & $-19,5$ & 1,2 & 3,2 \\
\hline MSR-A180 & Lagostomus maximus & $-17,7$ & 6,9 & 3,2 \\
\hline MSR-A252 & Lagostomus maximus & $-19,9$ & 8,1 & 3,2 \\
\hline MSR-A253 & Lagostomus maximus & $-13,6$ & 9,9 & 3,2 \\
\hline
\end{tabular}

\begin{tabular}{|c|c|c|c|c|}
\hline ID & Taxa & $\delta^{13} \mathrm{Ccol}$ & $\delta^{15} \mathrm{~N}$ & C:N \\
\hline MSR-A254 & Lagostomus maximus & $-21,9$ & 6,4 & 3,2 \\
\hline MSR-A255 & Lagostomus maximus & $-15,0$ & 7,9 & 3,2 \\
\hline MSR-A288 & Lagostomus maximus & $-18,1$ & 6,4 & 3,3 \\
\hline MSR-A182 & Myocastor coypus & $-23,7$ & 4,8 & 3,2 \\
\hline MSR-A366 & Myocastor coypus & $-23,2$ & 4,5 & 3,2 \\
\hline MSR-A292 & $\begin{array}{l}\text { Odontesthes } \\
\text { microlepidotus }\end{array}$ & $-16,7$ & 9,1 & 3,8 \\
\hline MSR-A293 & $\begin{array}{l}\text { Odontesthes } \\
\text { microlepidotus }\end{array}$ & $-21,1$ & 9,7 & 5,2 \\
\hline MSR-A334 & Oncifelis geoffroyi & $-17,8$ & 6,7 & 3,3 \\
\hline MSR-A134 & Percichthys sp. & $-13,5$ & 9,8 & 3,2 \\
\hline MSR-283 & Percichthys sp. & $-10,6$ & 10,1 & 2,7 \\
\hline MSR-284 & Percichthys sp. & $-9,5$ & 10,9 & 2,7 \\
\hline MSR-285 & Percichthys sp. & $-10,5$ & 10,4 & 2,7 \\
\hline MSR-288 & Percichthys sp. & $-11,2$ & 10,7 & 2,7 \\
\hline MSR-291 & Percichthys sp. & $-10,9$ & 11,2 & 2,7 \\
\hline MSR-292 & Percichthys sp. & $-11,5$ & 10,4 & 2,7 \\
\hline MSR-295 & Percichthys sp. & $-10,9$ & 10,1 & 2,7 \\
\hline MSR-A043 & $\begin{array}{l}\text { Phalacrocorax } \\
\text { olivaceus }\end{array}$ & $-11,3$ & 16,9 & 3,2 \\
\hline MSR-A346 & $\begin{array}{l}\text { Phoenicopterus } \\
\text { chilensis }\end{array}$ & $-12,5$ & 13,7 & 3,2 \\
\hline MSR-A347 & $\begin{array}{l}\text { Phoenicopterus } \\
\text { chilensis }\end{array}$ & $-12,6$ & 9,2 & 3,2 \\
\hline MSR-A353 & $\begin{array}{l}\text { Phoenicopterus } \\
\text { chilensis }\end{array}$ & $-12,1$ & 11,7 & 3,1 \\
\hline MSR-A355 & $\begin{array}{l}\text { Phoenicopterus } \\
\text { chilensis }\end{array}$ & $-14,5$ & 9,8 & 3,2 \\
\hline MSR-A356 & $\begin{array}{l}\text { Phoenicopterus } \\
\text { chilensis }\end{array}$ & $-12,7$ & 11,7 & 3,2 \\
\hline MSR-A365 & $\begin{array}{l}\text { Phoenicopterus } \\
\text { chilensis }\end{array}$ & $-13,8$ & 10,8 & 3,2 \\
\hline MSR-A251 & Lycalopex griseus & $-19,3$ & 8,1 & 3,2 \\
\hline MSR-A332 & Lycalopex griseus & $-20,8$ & 8,5 & 3,2 \\
\hline MSR-A354 & Lycalopex griseus & $-17,1$ & 6,2 & 3,1 \\
\hline MSR-A023 & Rhea americana & $-22,0$ & 5,9 & 3,2 \\
\hline MSR-A056 & Rhea americana & $-22,4$ & 6,2 & 3,2 \\
\hline MSR-A333 & Rheidae & $-21,0$ & 6,4 & 3,2 \\
\hline MSR-A179 & Serpentes & $-20,4$ & 7,7 & 3,2 \\
\hline MSR-A116 & Zaedyus pichiy & $-18,5$ & 9,9 & 3,4 \\
\hline MSR-A331 & Dasypodidae & $-16,1$ & 10,8 & 3,2 \\
\hline
\end{tabular}




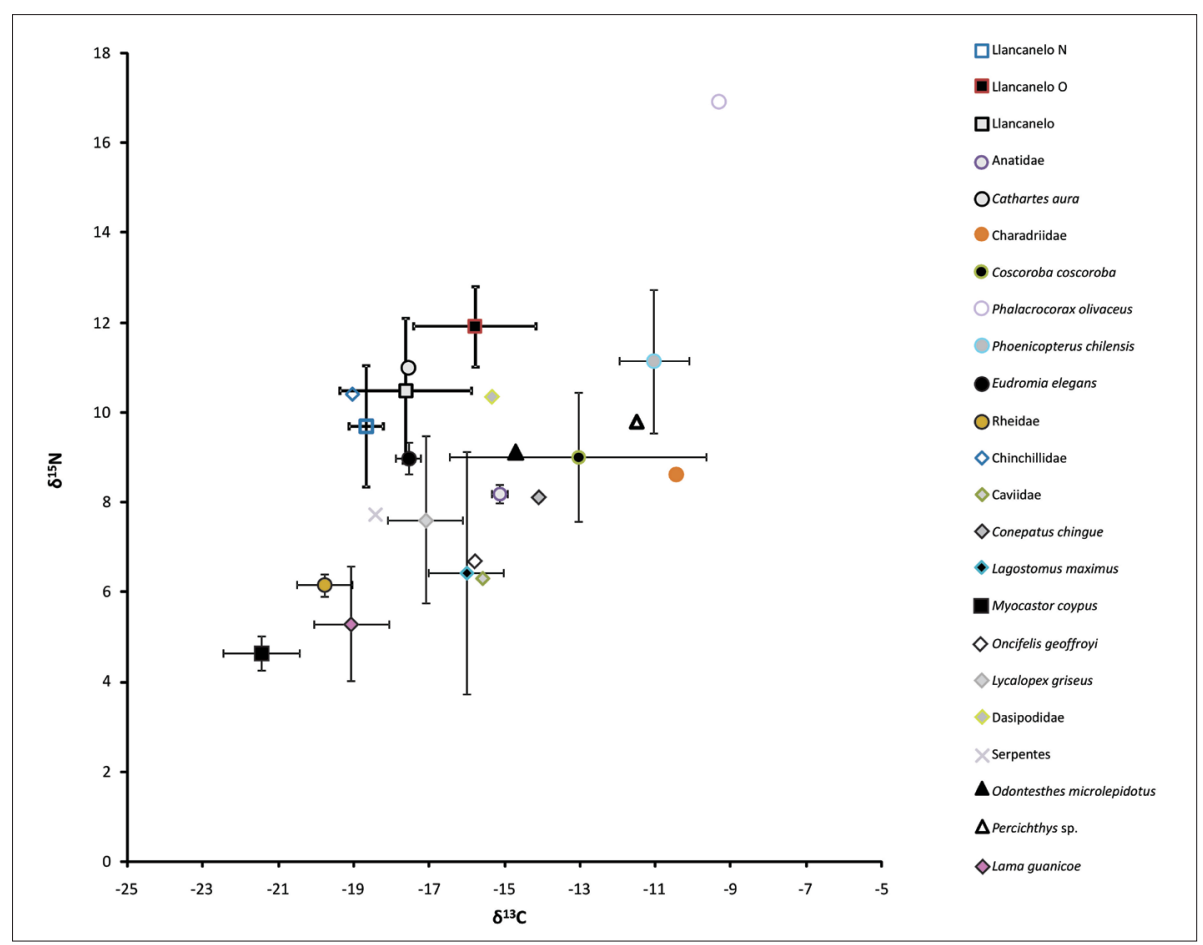

Figura 2. Promedios y desvío standard en los valores de $\delta^{13} \mathrm{C}$ y $\delta^{15} \mathrm{~N}$ para recursos y humanos de la región.

isotópico local los valores de $\delta^{15} \mathrm{~N}$ y $\delta^{13} \mathrm{C}$ también corresponden a un roedor, Myocastor coypus, con valores significativamente bajos para ambos isótopos $(4,6 \%$ y $-21,4 \%$ o respectivamente; Fig. 2).

Entre los peces, Percichthys sp. presenta valoresde $\delta^{13} \mathrm{C}$ significativamentemásenriquecidos que Odontesthes microlepidotus, con valores de $-10,8 \%$ y $-14,7 \%$ o respectivamente, pero ambos presentan similares valores promedios de $\delta^{15} \mathrm{~N}$ (aproximadamente 10\%o). Finalmente el único espécimen de serpiente presenta valores medios de $\delta^{15} \mathrm{~N}\left(7,7 \%\right.$ o) y empobrecidos en $\delta^{13} \mathrm{C}$ $(-18,4 \%$ o).

Tendencias en las muestras humanas

El conjunto de muestras humanas arqueológicas procedentes de Llancanelo (Fig. 1) provienen de la costa norte (7 individuos) y de la costa oeste (4 individuos). La Tabla 2 detalla la información cronológica, bioantropológica $e$ isotópica de estos individuos. Los mismos presentan variaciones en $\delta^{15} \mathrm{~N}$ entre $8 \%$ y $13 \%$, encontrándose una diferencia estadísticamente significativa entre las muestras del sector Norte y Oeste (Tabla 2; promedios 9,6\% y 11,9\%o respectivamente, one way ANOVA $\mathrm{F}=8,495$, $p=0,017)$. Los valores de $\delta 13 C$ col varían entre $-19,4 \%$ o y $-13,5 \%$ o con una diferencia significativa entre los sectores Norte y Oeste (Tabla 2, promedios $-18,66 \%$ o y $-15,78 \%$ o respectivamente, one way ANOVA $\mathrm{F}=20,5 \mathrm{p}=0,0014$ ); estos valores sobre la fracción hidroxiapatita varían $-15,9 \%$ o a $-7,7 \%$ o y también muestran diferencias significativas entre los sectores mencionados (promedios respectivos de $-14,30 \%$ y $-11,55 \%$, one way ANOVA $\mathrm{F}=7,45 \mathrm{p}=0,023)$. Finalmente los valores de $\delta^{18} \mathrm{O}$ varian entre $-1,7 \%$ o y $-8,8 \%$ o pero sin diferencias significativas entre ambos sectores (Tabla 2, one way ANOVA $F=0,8054 p=0,3929$ ).

Atendiendo los fechados ${ }^{14} \mathrm{C}$ de cada muestra humana (Tabla 2), se observan las tendencias temporales en $\delta^{15} \mathrm{~N}$ y $\delta^{13} \mathrm{C}$ (Fig. 3 y 4). La correlación Spearman entre tiempo y $\delta^{15} \mathrm{~N}$ es 0,68 $(p=0,020)$ mientras que la misma correlación entre tiempo y $\delta^{13} \mathrm{C}$ para colágeno es $0,51(p=0,10)$ en hidroxiapatita es de 0,50 ( $p=0,11)$. Esto indica 
Tabla 2. Características, cronología radiocarbónica y valores de isótopos estables de las muestras humanas analizadas.

\begin{tabular}{|c|c|c|c|c|c|c|c|c|c|c|c|c|}
\hline Muestra & Área & Sitio & Sexo & Edad & Código & $\begin{array}{l}{ }^{14} \mathrm{C} \text { años } \\
\mathrm{AP}\end{array}$ & $\begin{array}{c}\text { Código } \\
\text { USF }\end{array}$ & $\delta^{15} \mathrm{~N}$ & $\delta^{13} \mathrm{Ccol}$ & $\begin{array}{l}\text { Código } \\
\text { USF }\end{array}$ & $\delta^{13} \mathrm{Cap}$ & $\delta^{18} \mathrm{Oap}$ \\
\hline MRM-1 & \multirow{9}{*}{ 气ัँ } & $\begin{array}{l}\text { Llancanelo } \\
\text { Norte }\end{array}$ & M & $39-49$ & AA-62920 & $688 \pm 36$ & 8332 & 8 & $-19,4$ & 8361 & $-14,2$ & $-4,9$ \\
\hline MRM-7 & & $\begin{array}{l}\text { Llancanelo } \\
\text { Norte }\end{array}$ & $\mathrm{H}$ & $20-23$ & AA-62927 & $855 \pm 80$ & 8339 & 8,3 & $-19,1$ & 8368 & $-13,6$ & $-5,4$ \\
\hline MRM-5 & & $\begin{array}{l}\text { Llancanelo } \\
\text { Norte }\end{array}$ & $\mathrm{H}$ & $20-25$ & AA-62919 & $850 \pm 33$ & 8331 & 8,8 & $-18,8$ & 8360 & $-14,5$ & $-6,6$ \\
\hline MRM-25 & & $\begin{array}{l}\text { Llancanelo } \\
\text { Norte }\end{array}$ & - & A & AA-62926 & $900 \pm 36$ & 8338 & 9,9 & $-18,5$ & 8367 & $-14,1$ & $-5,5$ \\
\hline MRM-17 & & $\begin{array}{l}\text { Llancanelo } \\
\text { Norte }\end{array}$ & $\mathrm{H}$ & $20-25$ & AA-62934 & $1008 \pm 47$ & 8346 & 11,5 & $-18,3$ & 8375 & $-13,9$ & $-5,4$ \\
\hline MRM-23 & & $\begin{array}{l}\text { Llancanelo } \\
\text { Norte }\end{array}$ & & Juvenil & AA-62940 & $896 \pm 47$ & 8352 & 10,1 & $-18,3$ & 8381 & $-13,9$ & $-1,7$ \\
\hline \multirow[t]{3}{*}{ MRM-6 } & & $\begin{array}{l}\text { Llancanelo } \\
\text { Norte }\end{array}$ & & $4-5$ & AA-62925 & $862 \pm 33$ & 8337 & 11,1 & $-18,2$ & 8366 & $-15,9$ & $-5,7$ \\
\hline & & Promedio & - & - & - & - & - & 9,6 & $-18,6$ & - & $-14,3$ & $-5,03$ \\
\hline & & $\begin{array}{l}\text { Desvío } \\
\text { Estándar }\end{array}$ & - & - & - & - & - & 1,3 & 0,46 & - & 0,76 & 1,56 \\
\hline MRM-18 & \multirow{6}{*}{ ֻัँ } & $\begin{array}{l}\text { Los } \\
\text { Menucos }\end{array}$ & - & A & AA-62935 & $863 \pm 47$ & 8347 & 11,7 & $-17,3$ & 8376 & $-12,8$ & $-7,2$ \\
\hline MRM-14 & & $\begin{array}{l}\text { Pozos de } \\
\text { Carapacho }\end{array}$ & - & A & AA-62928 & $797 \pm 30$ & 8340 & 11,3 & $-15,8$ & 8369 & $-12,9$ & $-8,8$ \\
\hline AF-2081 & & $\begin{array}{l}\text { Cerro } \\
\text { Trapal }\end{array}$ & M & 19 a 21 & AA-66572 & $2626 \pm 61$ & 8359 & 11,4 & $-16,5$ & 8384 & $-12,8$ & $-5,3$ \\
\hline \multirow[t]{3}{*}{ AF-2077 } & & $\begin{array}{l}\text { Cerro } \\
\text { Trapal }\end{array}$ & M & 30 a 45 & AA-98700 & $2709 \pm 49$ & 8358 & 13,2 & $-13,5$ & 8383 & $-7,7$ & $-3,1$ \\
\hline & & Promedio & - & - & - & - & - & 11,9 & $-15,7$ & - & $-11,55$ & $-6,1$ \\
\hline & & $\begin{array}{l}\text { Desvío } \\
\text { Estándar }\end{array}$ & - & - & - & - & - & 0,8 & 1,64 & - & 2,57 & 2,4 \\
\hline
\end{tabular}

una relación fuerte $\mathrm{y}$ altamente probable entre el $\delta^{15} \mathrm{~N}$ y el tiempo pero baja probabilidad entre esta variable y $\delta^{13} \mathrm{C}$. Esta misma correlación entre los valores $\delta^{13} \mathrm{C}$ de colágeno y $\delta^{15} \mathrm{~N}$ es de 0,87 $(p=0,0004)$; para el sector Norte es de 0,93 ( $p=$ $0,0023)$ y para el sector Oeste es de $0,2(p=0,75)$ señalando una fuerte y altamente probable relación en los valores del Norte pero no del Oeste. Este test entre $\delta^{15} \mathrm{~N}$ y el $\delta^{13} \mathrm{C}$ de la hidroxiapatita da para todo el conjunto $0,67(p=0,02)$. Al evaluar por sector resulta para el sector Norte un $r s=-0,03$ $(p=0,95)$ y para el sector Oeste un $r s=0,94(p=$ $0,16)$. La correlación Spearman entre los valores $\delta^{13} \mathrm{C}$ de colágeno e hidroxiapatita es de 0,66 $(\mathrm{p}=$ $0,02)$. Al calcular esta variable por sector se obtuvo un $r s=-0,2(p=0,67)$ para el sector Norte y 0,31 $(p=1)$ para el sector Oeste.
Los valores de $\delta^{18} \mathrm{O}$ varian en los humanos entre $-1,7 \%$ a $-8,8 \%$ o V-PDB sin encontrarse diferencias estadísticamente significativas entre ambos sectores (Tabla 2; one way ANOVA $\mathrm{F}=0,8 ; \mathrm{p}=0,39)$. Muestras de agua analizada de la vertiente de Carapacho y pozos de Llancanelo (ambas de agua dulce) y la laguna en sector sur (salobre) dan valores diferentes $(-13,6 \%$ o, $-9,5 \%$ o y-7,2\%o V-SMOW respectivamente; ver detalle en Ugan et al. 2012). Usando la fórmula presentada en Gil et al. (2011, ver detalle en Knudson 2009) el valor promedio de $\delta^{18} \mathrm{O}$ de las muestras humanas se deriva de aguas bebidas con valores aproximados en -7,6\% V-SMOW y variando entre aproximadamente $-3,5 \%$ y $-13 \%$ o-SMOW. Usando el modelo presentado en Ugan et al. (2012) estos datos señalan que los individuos de 
Llancanelo provienen de residencias localizadas en distintos sectores de la región, incluyendo tanto las planicies como el piedemonte e incluso Payunia pero posiblemente con una señal más fuerte de esta última región, denominada en dicho modelo como Nevado-Payunia y donde se incluyen los datos hídricos de Llancanelo antes mencionados (Ugan et al. 2012: Fig. 3).

\section{Composición de la Dieta según SISUS}

La Tabla 3 resume los valores obtenidos según las simulaciones del software SISUS. Los porcentajes de biomasa promedio, mediana, y sus extremos están ajustados por las diferencias en la concentración y eficiencia en asimilación. Estos valores no son únicos. Por el contrario, ellos caracterizan una distribución de posibles soluciones para los datos entregados. Los resultados indican algunas diferencias y similitudes entre ambos sectores. En ambos casos la simulación señala una poca importancia de guanaco y reidos aunque pudieron ser más relevantes para los individuos enterrados en el Norte que en el Oeste. En ambos sectores los recursos faunísticos terrestres pequeños (entre estos incluimos armadillos, huevos de reidos, y roedores) fueron en términos de biomasa explotada más importantes que los antes mencionados, con valores medios entre $20-30 \%$. Los peces habrian tenido una escasa importancias (similar a guanaco y reidos) principalmente para los individuos del Norte pero casi el doble para los registrados en el Oeste. Otro de los recursos acuáticos, las aves, parecen haber aportado poco a la dieta de estos individuos. Finalmente es destacable el alto aporte de los vegetales, principalmente algarrobo pero también molle, que explican casi el $40-50 \%$ de la dieta. En ambos sectores el algarrobo fue muy importante mientras que el molle lo fue principalmente para los individuos del Norte pero no del Oeste.

\section{DISCUSIÓN}

La Fig. 2 muestra la relación isotópica de las muestras humanas de Llancanelo en los valores promedio de ambos isótopos, considerados tanto como un solo conjunto como segregados en sus dos sectores (Norte y Oeste). La misma figura también incluye estos parámetros tanto para los recursos con evidencias de explotación como para aquellos de los que se carece de tal evidencia. Las muestras humanas de Llancanelo se encuentran entre las de mayor valor de $\delta^{15} \mathrm{~N}$ de la región (Gil et al. 2011) y con valores de $\delta^{13} \mathrm{C}$ en colágeno que señalan

Tabla 3. Resultados de las dietas simuladas con SISUS (ver detalles en texto) para muestras humanas agrupadas por sector.

\begin{tabular}{|c|c|c|c|c|c|c|c|c|c|c|}
\hline 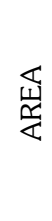 & & 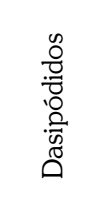 & 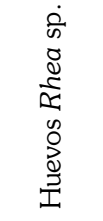 & 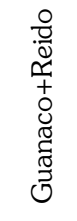 & $\begin{array}{l}\dot{0} \\
\hat{0} \\
\stackrel{2}{0} \\
\overline{0} \\
0 \\
0 \\
0\end{array}$ & 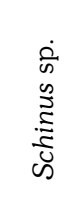 & 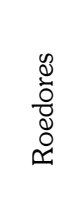 & 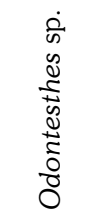 & 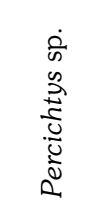 & $\begin{array}{l}\frac{0}{0} \\
\frac{0}{0}\end{array}$ \\
\hline \multirow{5}{*}{ 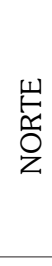 } & Media & $6 \%$ & $10,1 \%$ & $10 \%$ & $20,6 \%$ & $31 \%$ & $6 \%$ & $6 \%$ & $3 \%$ & $4 \%$ \\
\hline & DS & 0,05 & 0,05 & 0,07 & 0,09 & 0,14 & 0,05 & 0,05 & 0,03 & 0,03 \\
\hline & Min & 0 & 0 & 0 & 0 & 0 & 0 & 0 & 0 & 0 \\
\hline & Max & $36 \%$ & $20 \%$ & $48 \%$ & $51 \%$ & $76 \%$ & $41 \%$ & $37 \%$ & $23 \%$ & $25 \%$ \\
\hline & Mediana & $4,8 \%$ & $11,2 \%$ & $8,1 \%$ & $20,5 \%$ & $32 \%$ & $5 \%$ & $5,3 \%$ & $2,9 \%$ & $3,2 \%$ \\
\hline \multirow{5}{*}{ 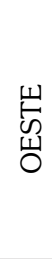 } & Media & $17,8 \%$ & $3 \%$ & $3,8 \%$ & $27 \%$ & $9,6 \%$ & $5 \%$ & $12,2 \%$ & $11 \%$ & $8,7 \%$ \\
\hline & DS & 0,12 & 0,02 & 0,03 & 0,07 & 0,07 & 0,04 & 0,10 & 0,08 & 0,07 \\
\hline & Min & 0 & 0 & 0 & 0 & 0 & 0 & 0 & 0 & 0 \\
\hline & Max & $73 \%$ & $16 \%$ & $21 \%$ & $47 \%$ & $51 \%$ & $30 \%$ & $70 \%$ & $45,7 \%$ & $52 \%$ \\
\hline & Mediana & $15,4 \%$ & $2,5 \%$ & $3 \%$ & $28,2 \%$ & $7,9 \%$ & $4 \%$ & $9,5 \%$ & $10,1 \%$ & $6,6 \%$ \\
\hline
\end{tabular}


una dieta con proteínas principalmente generadas sobre recursos $\mathrm{C}^{3}$. La fracción apatita confirma este mismo patrón para la fracción energética de la dieta. Los valores de nitrógeno están elevados más de 3\%o respecto a los carnívoros de Llancanelo y son más altos que varios recursos acuáticos (Fig. 2). Esto señalaría un componente de estos últimos recursos en la dieta humana de Llancanelo que también es confirmada por la alta correlación entre $\delta^{13} \mathrm{Ccol}$ y $\delta^{15} \mathrm{~N}$. Analizadas estas muestras por sector, se nota que los valores más altos de $\delta^{15} \mathrm{~N}$ se registran predominantemente en el Oeste, mientras que también estas muestras están más enriquecidas en $\delta^{13} \mathrm{C}$ (ver en Resultados). Es decir, las muestras del Oeste reflejarían un mayor componente de recurso acuático que las del Norte. Pero ambos conjuntos presentan valores de $\delta^{13} \mathrm{C}$ más negativos que los esperables por un consumo importante de estos recursos y quedan comprendidos entre los valores de los acuáticos y de los terrestres (principalmente Lama guanicoe y Rheidae). De allí la importancia de SISUS al posibilitar una evaluación estadística de ambos isótopos considerando múltiples fuentes para la dieta de estos individuos.

Nuestra propuesta no es entender los valores de SISUS en términos absolutos sino, al menos en esta instancia exploratoria, usarlos como contrapeso a nuestras inferencias arqueológicas. Por ello creemos que los modelos de mezcla isotópica (una revisión en Boecklen et al. 2011) dan una perspectiva en una escala nominal que luego podemos discutir con el resto del registro arqueológico. Entendiendo esto, presentamos las tendencias obtenidas. Los valores medios de SISUS señalan que los recursos acuáticos, incluyendo tanto ambos taxones de peces como patos, nunca habrían sido mayoritarios en las dietas humanas de Llancanelo, aunque parecen haber sido más significativos para las muestras del sector Oeste que del Norte. En ningún caso (incluso simulaciones con otras variables y especies que aquí no se presentan) el grupo "guanaco+reido" fue importante, y nunca superó el valor medio del $10 \%$. Eso es importante al menos para entender las estrategias humanas en las tierras bajas. En cambio, los recursos pequeños (incluyendo aquí los grupos "dasipódidos", "huevos reido" y "roedores") habrían sido más relevantes que "guanaco-reido", generalmente en el orden del 20 a $30 \%$. En ambos sectores se confirma la fuerte importancia que habría tenido en la dieta humana el algarrobo, generalmente mayor que la de los recursos pequeños recién mencionados, a lo que también se suman los molles pero principalmente para las muestras del Norte. Sin dudas, estas simulaciones sirven para alertar de escenarios pero no son una definición específica de estas dietas. Entre otras razones porque están limitadas a nuestra información sobre las especies explotadas. Cualquier taxón que hayamos ignorado, o que erróneamente consideráramos como explotados por los humanos, generará un modelo más alejado de la situación posible.

Los sitios arqueológicos Llan 2, Llan 17, Llan 22, Llan 29, y Llan 50 ofrecen información sobre los recursos explotados (Tabla 4; Giardina et al. 2012; Corbat et al. 2009; Corbat 2012). Ellos se localizan próximos a la laguna actual, en una distancia siempre menor a $5 \mathrm{~km}$ (Fig. 1). Los sitios Llan 29 y Llan 50, próximos entre ellos, se emplazan en el Oeste de la laguna y muy cercanos a pozos de agua dulce (Pozos Llancanelo o Carapacho). Los otros sitios se emplazan en el Norte de la laguna, siendo Llan 17 el único muy cercano a una fuente de agua dulce, como el Arroyo Malo (Fig. 1). La Tabla 4 detalla la superficie excavada y las cronologías radiocarbónicas disponibles para estos conjuntos. También esa tabla incluye los valores NISP para cada categoría taxonómica, excepto para huevos que se detalla en gramos. En esta tabla se destaca el escaso registro de camélidos. Esta familia se registró sólo en sitios del Oeste, mostrando para los últimos 3.000 años 3 especímenes en 2 sitios (Tabla 4). Sumado a esto, no se registró material óseo identificado como Rhea sp. sino sólo en fragmentos de huevos. Esta situación es concordante con lo inferido por el modelo SISUS. También, en una escala mayor, es concordante con el registro zooarqueológico de las tierras bajas (Gil 2006; Neme \& Gil 2008; Giardina 2012; Otaola et al. 2012). En todos los sitios de Llancanelo se registraron una cantidad y diversidad significativa de recursos pequeños (incluyendo huevos de reidos, dasipódidos, aves pequeñas y roedores). Sobre el registro zooarqueológico de peces y patos, es notoria su mayor abundancia en el Oeste que en el Este, incluso cuando agrupadamente el primero tiene superficies excavadas menores 
(Tabla 4). Estas dos situaciones, la abundancia de estos grupos y su diferencia entre los sectores, también son coincidentes con las tendencias simuladas por los valores medios obtenidos en SISUS. Sin embargo, el algarrobo, considerado por SISUS como uno de los recursos de mayor aporte en estas muestras, no se ha registrado en los sitios de Llancanelo, a pesar de encontrarse actualmente en el área (Llano 2012) y haber sido una de las especies más explotadas en las tierras bajas vecinas (Llano \& Andreoni 2012). Esto no debilita la inferencia de SISUS. Por una parte el registro de manos de moler y molinos en Llancanelo puede indicar la explotación de estos recursos vegetales que, por historias tafonómicas o muestreos, no hay entre las evidencias arqueobotánicas. Por otra parte, ya que el registro isotópico promedia la historia de vida, esta discordancia puede señalar, alternativamente, la conexión de estos individuos con otros sectores donde estos recursos sí fueron más abundantes y claramente registrados arqueológicamente.

Los registros zooarqueológicos más antiguos provienen del sector Oeste, con materiales desde ca. 3.200 años AP, mientras que para el Este se limita a los últimos 1.000 años AP. El registro de guanacos sólo se localiza en el Oeste y es notoriamente mayor la densidad temporal de peces

Tabla 4. Abundancia taxonómica (NISP) por sitio en Llancanelo. Los restos de Rhea ssp. corresponden sólo a cáscaras de huevos y están cuantificados en gramos, señalándose sus valores entre paréntesis. Las cantidades de placas dérmicas de dasipódidos están entre corchetes.

\begin{tabular}{|c|c|c|c|c|c|c|}
\hline \multirow[b]{2}{*}{ Taxón } & \multicolumn{3}{|c|}{ SECTOR NORTE } & \multicolumn{3}{|c|}{ SECTOR OESTE } \\
\hline & Llan 2 & Llan 17 & Llan 22 & Llan 29 & Llan 50 Sup. & Llan 50 Inf. \\
\hline Indet. & - & - & - & - & 10 & 1 \\
\hline Reptil indet. & - & - & - & 2 & 4 & 9 \\
\hline Peces indet. & - & 21 & 1 & 42 & 396 & 223 \\
\hline Odontesthes microlepidotus & - & 2 & - & 48 & 298 & 186 \\
\hline Percichtys sp & - & 2 & - & - & - & - \\
\hline Ave indet. & - & - & - & - & - & 1 \\
\hline Ave chica indet. & - & - & - & - & - & 2 \\
\hline Ave mediana indet. & $0(1,76)$ & 1 & $1(1,76)$ & $22(7,76)$ & $16(3,34)$ & $23(5,12)$ \\
\hline Rhea ssp. & $(15,29)$ & - & $(1,33)$ & $(76,37)$ & $(19,7)$ & $(39,76)$ \\
\hline Anatidae indet. & - & - & 1 & 5 & 13 & 13 \\
\hline Rallidae indet. & - & - & 1 & 4 & - & 1 \\
\hline Charadiidae indet. & - & - & - & - & 1 & 1 \\
\hline Passeriforme indet. & - & - & - & - & - & 1 \\
\hline Dasipodidae indet. & $2[143]$ & - & 1 [9] & 8 [172] & $3[64]$ & $4[56]$ \\
\hline Lama guanicoe & - & - & - & 3 & - & 1 \\
\hline Herbivora indet. & - & - & - & - & 7 & 1 \\
\hline Mammalia indet. & - & - & - & - & 11 & 2 \\
\hline Micromammalia indet. & - & 2 & 2 & 58 & 20 & 69 \\
\hline Mammalia grande indet. & - & - & - & 2 & - & 1 \\
\hline Mammalia mediano indet. & 3 & - & 1 & 17 & 39 & 13 \\
\hline Mammalia mediano/chico indet. & - & - & - & 6 & 3 & 1 \\
\hline Mammalia mediano/grande indet. & - & - & - & - & - & 1 \\
\hline Vertebrata chico indet. & - & - & - & - & - & 1 \\
\hline Vertebrata mediano indet. & - & - & - & - & 29 & 16 \\
\hline Artiodactyla indet. & - & 1 & - & 2 & - & - \\
\hline Superficie de la muestra $\left(\mathrm{m}^{2}\right)$ & 0,25 & 4 & 0,25 & 2 & 2 & 2 \\
\hline CRONOLOGÍA (años AP) & - & $990 \pm 34$ & $798 \pm 36$ & $3039 \pm 28$ & $553 \pm 34$ & $3199 \pm 38$ \\
\hline
\end{tabular}


en este sector en relación al Norte (Corbat 2012). Estas diferencias complementan la tendencia observada en los isótopos y cronologías de las muestras humanas de Llancanelo. El sector Oeste tiene los restos humanos más antiguos y, entre otras diferencias isotópicas, aquí se registran los valores de $\delta^{15} \mathrm{~N}$ más altos respecto al Norte. En ese sentido, en la escala de la laguna, los resultados muestran una importante variabilidad vinculada a la subsistencia, tanto en los resultados de isótopos como en el registro zooarqueológico, en la escala de la laguna.

\section{Dieta Humana de Llancanelo} en el contexto del Sur de Mendoza

Con la intención de evaluar la homogeneidad de las dietas de Llancanelo respecto a la región, se comparan las mismas con resultados previos obtenidos sobre muestras que aquí se agrupan en las áreas de Cordillera, Piedemonte, Planicies Orientales y La Payunia (Fig. 3; para una definición de estas unidades espaciales ver Neme \& Gil 2012). Estas zonas del sur de Mendoza son definidas en base a diferencias fitogeográficas, ecológicas y climáticas que implican patrones fotosintéticos distinguibles isotópicamente entre ellas (dominancias de $\mathrm{C}^{3}$ o $\mathrm{C}^{4}$ y CAM) y por ello permiten discutir rangos de acción y movilidad humana (Gil et al. 2012). Primero se evalúan las tendencias en cada isótopo y luego la combinación de ambos en los mencionados conjuntos. Finalmente se comparan los individuos según la relación entre los valores de $\delta^{13} \mathrm{C}$ de colágeno e hidroxiapatita.

Se observa en el conjunto humano de Llancanelo una dieta isotópica similar a la del resto del sur de Mendoza; así no se encontraron diferencias estadísticas (usando one way ANOVA y Mann-Whitney) en los valores de $\delta^{15} \mathrm{~N}$ de Llancanelo respecto a los de las restantes zonas.

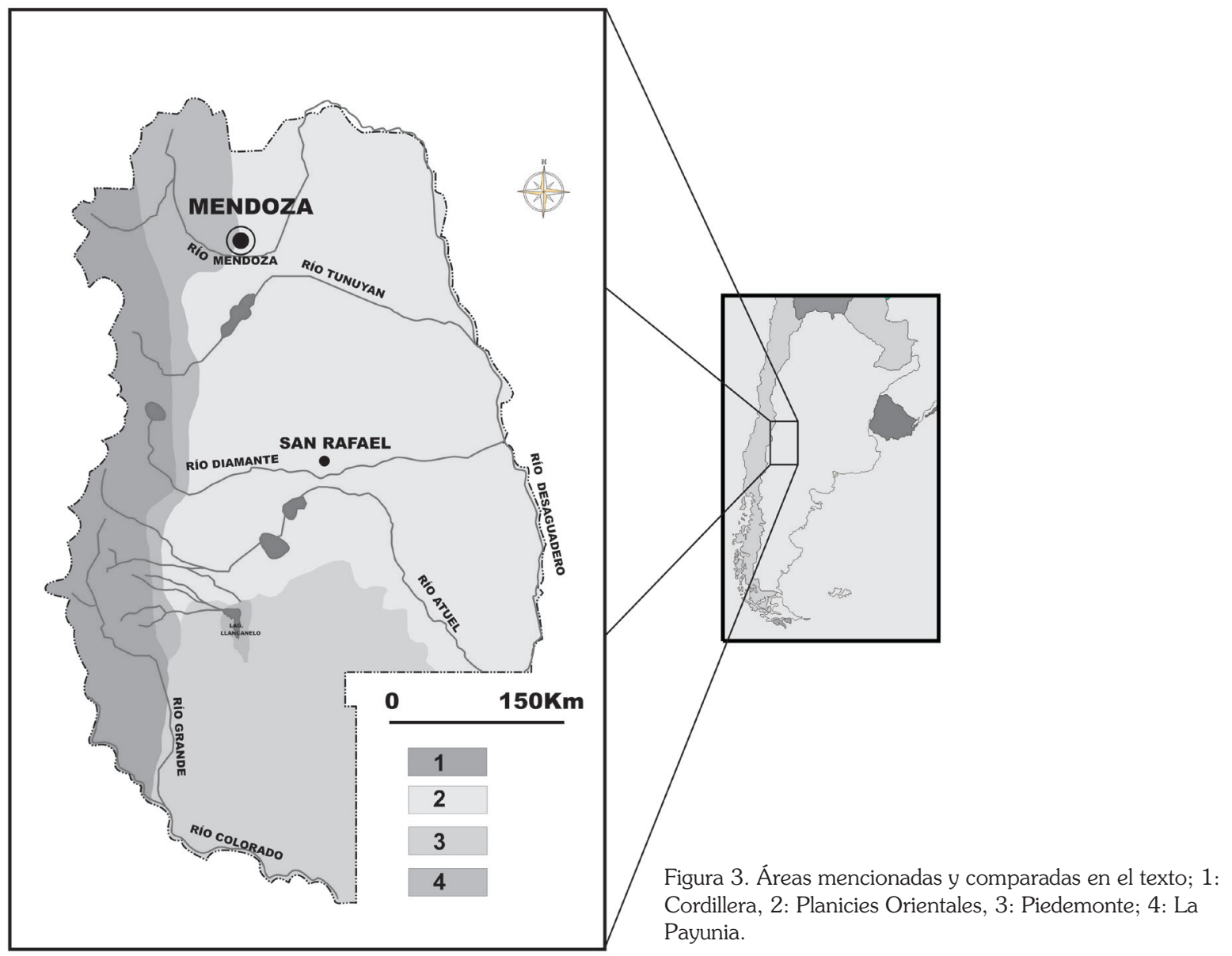


Previamente habíamos interpretado los valores de este isótopo en Llancanelo como un reflejo del consumo de recursos acuáticos; esta similitud con el resto del sur de Mendoza debilita esa tendencia o, por el contrario, extrapola la explotación de esos recursos a toda la región. Esta última opción es poco sostenida por la zooarqueología en esa escala espacial. También deberán evaluarse otros recursos en el sur de Mendoza que tengan una señal isotópica similar a la de los peces de Llancanelo. Por su parte los valores de $\delta^{13} \mathrm{C}$ en colágeno de Llancanelo se diferenciaron sólo de Planicie Oriental (donde están más enriquecidos) pero no se diferenciaron en forma significativa de las otras zonas. Esta diferencia con Planicie Oriental puede deberse a que las poblaciones de esta última se enmarcan en la región fitogeográfica del Monte, donde las plantas $\mathrm{C}^{4}$ son más abundantes que en las otras regiones del sur mendocino y a que allí parece haber sido más clara la incidencia del maíz en las dietas prehispánicas, lo que potenciaría aún más el mencionado enriquecimiento (Gil \& Neme 2010; Gil et al. 2011). Estudios previos en dieta de camélidos y sobre huevos de reidos confirman también esta separación del Monte (básicamente Planicie Oriental) respecto a otras áreas en términos de $\delta^{13} \mathrm{C}$ (Gil et al. 2012; Giardina et al. 2013) lo que señala que las diferencias fitogeográficas habrían impactado en la dieta de estas especies.

$\mathrm{Al}$ analizar por sectores, las tendencias en $\delta^{15} \mathrm{~N}$ del Norte muestran similitud con los valores de las Planicies Orientales, Cordillera y Piedemonte; pero se diferencian en forma significativa de Payunia; contrariamente las muestras del Oeste son estadísticamente similares a las de Payunia pero se diferencian significativamente de los otros sectores (Fig. 4). Realizada esta comparación con las tendencias en $\delta^{13} \mathrm{Ccol}$ se encontraron diferencias significativas entre las muestras del sector Norte con todas las áreas. Pero el sector Oeste no se diferenció de los resultados obtenidos en ellas. Confirmando esta tendencia, los valores de $\delta^{13} \mathrm{C}$ obtenidos en hidroxiapatita señalan que la muestra del Norte se diferencia de todos los sectores del sur de Mendoza mientras que el Oeste no se diferencia de ninguno, confirmando la tendencia previa. Entonces la fracción proteica $\left(\delta^{13} \mathrm{Ccol}\right)$ y energética $\left(\delta^{13} \mathrm{Cap}\right)$ de la dieta en el sector Oeste es similar a la del resto del sur de
Mendoza; mientras que las muestras del Norte se diferencia a las del área, siendo en general más empobrecidas en ambas fracciones del mencionado isótopo. Si se analiza como un solo conjunto, los valores de Llancanelo son similares a los de toda la región, excepto a las muestras más enriquecidas de Planicie Oriental.

Si para la comparación Llancanelo-Sur de Mendoza se combinan ambos isótopos usando los valores medios y desvío estándar se observa la tendencia de la Fig. 4. Los patrones de $\delta^{15} \mathrm{~N}$ y $\delta^{13} \mathrm{C}$ de los conjuntos del sur de Mendoza colocan a Llancanelo Norte y Oeste en extremos diagonales opuestos dentro de las tendencias regionales. Por un lado los valores humanos del Norte de Llancanelo representan los promedios más negativos en $\delta^{13} \mathrm{Ccol}$ y más bajos de $\delta^{15} \mathrm{~N}$ mientras que los resultados del Oeste señalan la tendencia más enriquecida y nitrógenos más elevados de la región (Fig. 4). Asimismo las muestras del Norte se agrupan más próximas a las tendencias de Cordillera y Piedemonte, mientras que las del Oeste lo hacen principalmente con Payunia y, en menor grado, Planicie Oriental. Es notoria la diferencia en $\delta^{15} \mathrm{~N}$ que presentan las muestras del Oeste respecto a los otros sectores del sur mendocino y que se asemejan a la tendencia de Payunia (Fig. 4). Estos valores están por encima de los carnívoros de Llancanelo, lo que podría explicarse como explotación de recursos acuáticos, como anteriormente se ha señalado. Sin embargo, las muestras de Payunia no pueden explicarse fácilmente por estos recursos salvo que en el pasado existiera una fuerte conexión entre estos sectores contiguos, tendencia que es apoyada por los datos de $\delta^{18} \mathrm{O}$. Los datos presentados en Gil et al. (2011) y Ugan et al. (2012) señalan a las muestras humanas de Payunia diferenciadas del resto del sur de Mendoza; y también se muestran estadísticamente similares a las de Llancanelo. La otra explicación para estos altos valores de $\delta^{15} \mathrm{~N}$ podría ser el estrés a la aridez pero en ese sentido es difícil sostener que este estrés lo sufrieran las muestras de Llancanelo Oeste y Payunia pero no las de Planicie Oriental, por ejemplo, ya que están sometidas a tendencias climáticas semejantes. Así mismo el registro zooarqueológico de Llancanelo señala una frecuencia alta de restos de peces que serían producto de su consumo (Corbat 2012). 


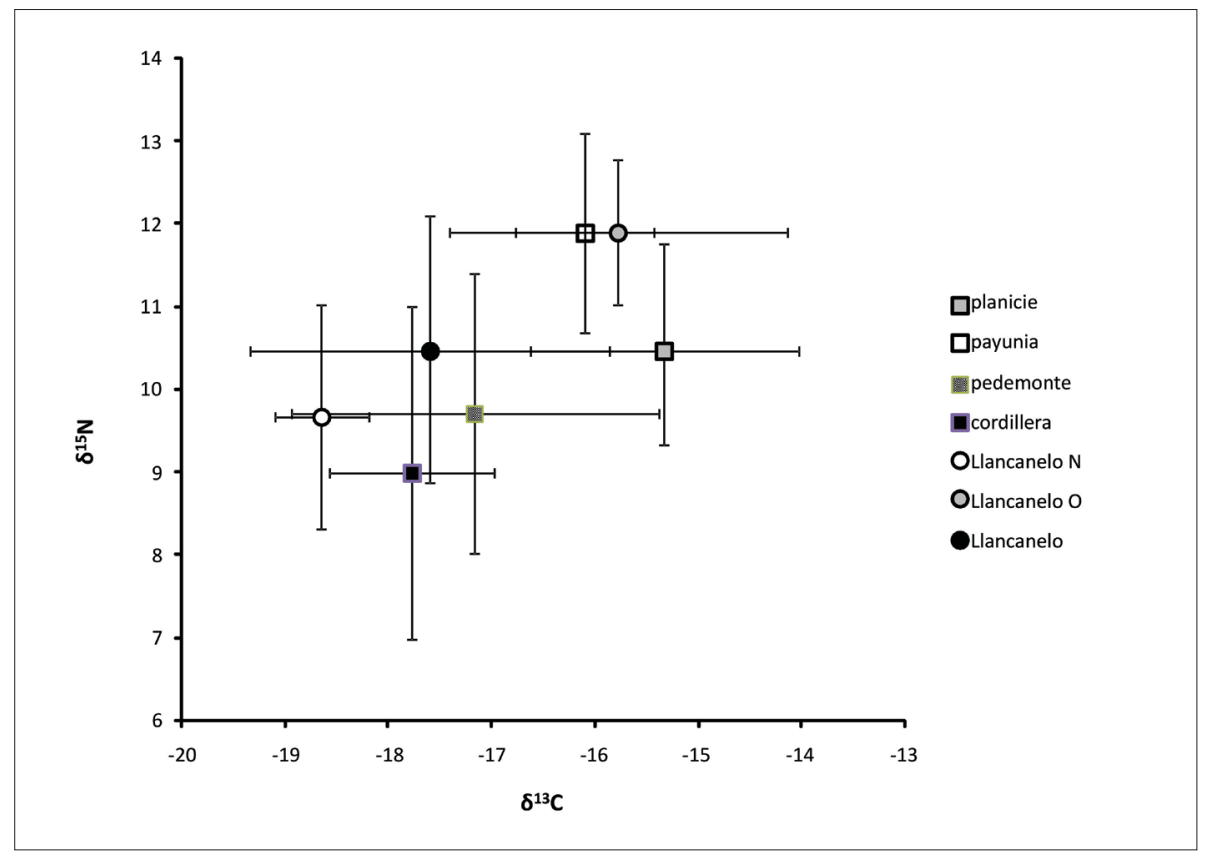

Figura 4. Tendencias en los promedios y desvío estándar en los valores de $\delta^{13} \mathrm{C}$ y $\delta^{15} \mathrm{~N}$ de las muestras humanas del sur de Mendoza agrupadas por sector ambiental (ver detalles en texto).

Visto como conjunto el valor medio de las dietas humanas de Llancanelo se asemeja a Cordillera y Piedemonte pero considerando su variación, muestra vínculos con la Planicie Oriental y Payunia.

Comparando los valores individuales de $\delta^{13} \mathrm{C}$ en colágeno e hidroxiapatita se observa en la Fig. 5 que las muestras del sector Oeste se agrupan principalmente junto a las de Payunia, Piedemonte y Planicie Oriental, mientras que las del sector Norte se agrupan junto a las de Piedemonte. Ambos conjuntos no se superponen en el mencionado modelo. Sobre esa misma figura, y usando el mencionado modelo Kellner y Schoeninger (2007), se infiere que la dieta humana de Llancanelo está basada principalmente en proteínas y energías $\mathrm{C}^{3}$, con excepción del individuo AF-2077 cuyas fuentes están significativamente más basadas en $\mathrm{C}^{4}$ estando entre las muestras más enriquecidas del sur de Mendoza y teniendo la misma una fecha de 2.700 años ${ }^{14} \mathrm{C}$ (Tabla 2). En este caso es más sencillo explicar este patrón por el consumo de otros recursos diferentes al maíz, principalmente por su cronología y contexto, y entre los que podríamos incluir los acuáticos como los peces (ver Fig. 2).
Volviendo a nuestra cuestión sobre la homogeneidad de las dietas humanas en Llancanelo respecto al sur de Mendoza, la respuesta merece dos escalas de análisis. La primera escala, comparando Llancanelo como un solo conjunto con el resto de los conjuntos del sur de Mendoza; y una segunda escala, diferenciando cada sector (Norte y Oeste) y comparándolos con el resto de las muestras del sur de Mendoza.

Analizadas como un solo grupo, las muestras humanas de Llancanelo no tienen una dieta diferente, estadísticamente significativa, al resto del sur de Mendoza. Esto implicaría una articulación de estas poblaciones de la laguna con los distintos sectores de la región. También rechaza la idea de una segregación de estas poblaciones, con una adaptación fuerte a estos espacios lacustres que las diferenciaría de los otros sectores. Si los recursos acuáticos tuvieron algún rol en Llancanelo, éste no fue significativamente fuerte como para diferenciarlos del resto de la región o en promedio dicho recurso compuso parte de las dietas en las muestras registradas en el sur de Mendoza.

$\mathrm{Si}$ las tendencias de Llancanelo se observan por sectores, las muestras del sector Oeste muestran valores semejantes a los de Payunia, y 


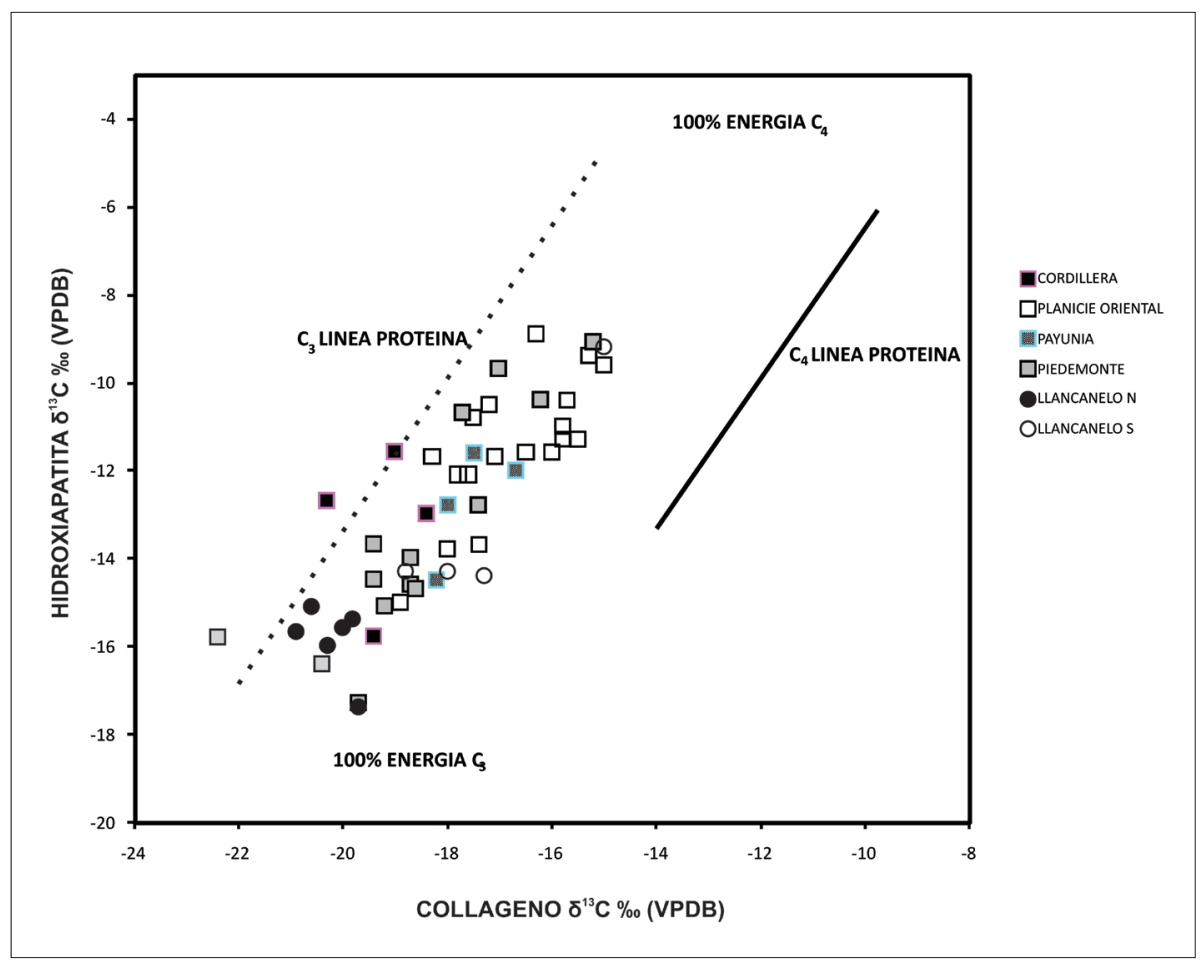

Figura 5. Relación de los valores $\delta^{13} \mathrm{C}$ en hidroxiapatita y colágeno de cada individuo por región y usando como marco de referencia al modelo de Kellner y Schoeninger (2007).

Planicie Oriental; mientras que los del sector Norte se asemejan a los de Piedemonte. Esta tendencia abre la posibilidad de entender a la laguna como un espacio articulador de diferentes esferas o rangos de acción. Por un lado, y principalmente para las muestras más antiguas, un vínculo de la laguna con Payunia y Planicie Oriental; mientras que por el otro se nota, en muestras principalmente post $1.000 \mathrm{AP}$, un vínculo de la laguna con el Piedemonte.

\section{CONCLUSIONES}

Este trabajo buscó caracterizar la dieta humana de individuos registrados en este humedal para luego discutir la similitud o diferencia con el resto del sur de Mendoza. Los resultados señalan en general una fuerte importancia de recursos pequeños y de ambientes acuáticos. Llamativamente tanto el registro zooarqueológico como la simulación de dieta isotópica con SISUS señalan una baja o nula importancia de guanaco/ reidos (cabe mencionar que para este recurso no se incluyen los huevos que sí parecieron ser importantes). En términos generales, los isótopos no diferencian estas dietas del resto del sur de Mendoza, lo que podría señalar un fuerte promedio de las mismas que se explicarían por una movilidad generalizada. Pero internamente, sí hay variaciones entre las dietas y los registros del Norte y Oeste de Llancanelo. Los individuos del Norte señalan una semejanza isotópica con el Piedemonte mientras que los del Oeste lo hacen con Payunia y Planicie Oriental. La laguna parece, en ese sentido, ser un nodo articulador de las poblaciones que ocuparon el sur de Mendoza, por ello no parece ser un espacio circunscripto para el uso restringido de poblaciones en escala local. Esta tendencia también es concordante con los valores de $\delta^{18} \mathrm{O}$ de las muestras humanas aquí presentadas.

\section{AGRADECIMIENTOS}

Versiones previas de este trabajo se presentaron en las VIII Jornadas de Arqueología de la Patagonia (Malargüe 2011), y en el "II Encuentro Latinoamericano de Zooarqueología" 
(Santiago de Chile 2012). Los trabajos de campo y análisis de laboratorio se realizaron con el apoyo económico de CONICET, Agencia Nacional de Promoción Científica y Tecnológica, la Universidad Nacional de Cuyo y National Science Foundation. Agradecemos las observaciones y sugerencias de dos evaluadores. También a la Dirección Provincial de Recursos Naturales Renovables (Gobierno de Mendoza), específicamente a su personal administrativo de Malargüe y a los guardaparques de Llancanelo. Ellos mostraron interés por estos trabajos y realizaron aportes muy valiosos. Los pobladores locales de Llancanelo, específicamente las familias Pardo, Pérez y Sagal, dieron información muy útil para este estudio y también aportaron muestras para los análisis de isótopos estables. El Museo Regional de Malargüe permitió tomar muestras óseas pequeñas que fueron fundamentales para definir las dietas humanas y su cronología radiocarbónica. Finalmente queremos recordar a Don Jorge Luna, con quien tuvimos el honor de realizar algunas caminatas por las playas y coladas de este increíble humedal.

\section{BIBLIOGRAFÍA}

Ambrose, S. H. (1990). Preparation and Characterization of Bone and Tooth Collagen for Isotopic Analysis. Journal of Archaeological Science 17, 431-451.

(1993). Isotopic Analysis of Paleodiets: Methodological and Interpretive Considerations. En Sandford, M. (Ed), Investigations of Ancient Human Tissue (pp. 59-129). New York: Gordon and Breach Science Publishers.

Ambrose S., y De Niro, M. (1986). Recontruction og African human diet using bone collagen carbon and nitrogen isotope ratios. Nature 319, 321-324.

Ambrose S. y Norr, L. (1993). Experimental evidence for the relationship of the carbon isotope ratios of whole diet and dietary protein to those of bone collagen and carbonate. En J. Lambert y G. Grupe (Eds.), Prehistoric human bone: Archaeology at the molecular level (pp. 1-37). Berlin: Springer-Verlag.

Barberena, R. (2002). Los límites del mar. Isótopos estables en Patagonia Meridional. Buenos Aires: Sociedad Argentina de Antropología.

(2013). Biogeografía, competencia y demarcación simbólica del espacio: modelo arqueológico para el norte de Neuquén. Intersecciones en Antropología 14: 367381.
Bocherens, H. y Drucker, D. (2003). Reconstructing Neandertal diet from 120.000 to 30.000 BP using carbon and nitrogen isotopic abundances. En M., Patou-Mathis y H. Bocherens (Eds.), Le Role de l'Environnement dans les Comportements des Chasseurs-Cueilleurs Pre historiques (pp. 1-7). Oxford: British Archaeological Reports International Series 1105, Archaeopress.

Boecklen, W. J., Yarnes, C. T., Cook, B. A., y James, A. C. (2011). On the use of stable isotopes in trophic ecology. Annual Review in Ecology and Systematics 42, 411-440.

Bonnat, F. y Franchetti, F. (2010). Tendencias distribucionales del registro lítico y cerámico en Llancanelo (Malargüe, Mendoza). Mendoza: Trabajo presentado en el XVII Congreso Nacional de Arqueología Argentina.

Buzon, M. R., Conlee, C. A., y Bowen, G. J. (2011). Refining oxygen isotope analysis in the Nasca region of Peru: an investigation of water sources and archaeological samples. International Journal of Osteoarchaeology 21(4), 446-455.

Coltrain, J., y Leavitt, S. (2002). Climate and diet in Fremont prehistory: Economic variability and abandonment of maize agriculture in the Great Salt Lake basin. American Antiquity 67, 453-485

Corbat, M. (2012). Una aproximación tafonómica a los restos de peces en Laguna Llancanelo (Mendoza). MS.

Corbat M., Zangrando, F. A., y Gil, A. (2009). El Estudio de Restos de Peces en Conjuntos Arqueológicos del Sur de Mendoza. En M. Salemme, F. Santiago, M. Álvarez, E. Piana, M. Vázquez y M. E. Mansur (Eds.), Arqueología de Patagonia: una mirada desde el último confín (pp. 717-728). Ushuaia: Editorial Utopías.

Dansgaard, W. (1964). Stable isotopes in precipitation. Tellus 16 (4), 436-468

Dupras, T. y Schwarcz, H. (2001). Strangers in a strange land: stable isotope evidence for human migration in the Dakhleh Oasis, Egypt. Journal of Archaeological Science 28(11), 1199-1208.

Erhardt, E. B. (2007). Stable Isotope Sourcing using Sampling. Software, CRAN R package "sisus".

(2008). SISUS: Stable Isotope Sourcing using Sampling, Getting Started, R package.

(2009). Stable Isotope Sourcing using Sampling. New Mexico, Albuquerque: Dissertation, University of New Mexico.

Fogel, M. L., y Tuross, N. (2003). Extending the limits of paleodietary studies of humans with compound specific carbon isotope analysis of amino acids. Journal of Archaelogical Sciene 30, 535-545.

Friedli H., Lötscher, H., Oeschger, H., Siegenthaler, U., 
y Stauffer, B. (1986). Ice core record of the ${ }^{13} \mathrm{C} /{ }^{12} \mathrm{C}$ ratio of atmospheric $\mathrm{CO}_{2}$ in the past two centuries. Nature 324, 237-238.

Fry, B. (2008). Importance of open bays as nurseries for Louisiana brown shrimp. Estuaries and Coasts 31:776-789.

Gat, J. R. (1996). Oxygen and hydrogen isotopes in the hydrologic cycle. Annual Review of Earth and Planetary Sciences 24, 225-262.

Giardina, M. (2012). Intensificación en el sur de Mendoza: un enfoque avifaunístico. Archaeofauna 21, 207-222.

Giardina M, Neme, G., Corbat, M., Ugan, A., Otaola, C., y Gil, A. (2012). Tendencias isotópicas y arqueofaunísticas en Laguna LLancanelo (Mendoza, Argentina). Santiago de Chile: II Encuentro Latinoamericano de Zooarqueologia.

Giardina M., Neme, G., y Gil, A. (2013). Rheidae Egg Human Exploitation and Stable Isotopes: Trends from West Central Argentina. International Journal of Osteoarchaeology. DOI: 10.1002/oa.2346.

Gil, A. F. (2006). Arqueología de La Payunia (Mendoza, Argentina). El poblamiento humano en los márgenes de la agricultura. Oxford: BAR International Series 1477.

Gil, A., y Neme, G. (2006). Distribuciones arqueológicas superficiales en Payunia-Llancanelo. Anales de Arqueología y Etnología 61, 163-184.

(2010). Registro arqueológico en la cuenca media del Atuel: viejos y nuevos problemas; viejos y nuevos datos.

En M. Zárate, A. Gil y G. Neme (Eds.), Condiciones paleoambientales y ocupaciones humanas durante la transición Pleistoceno-Holoceno y Holoceno de Mendoza (pp. 239-276). Buenos Aires: Sociedad Argentina de Antropología.

Gil, A., Neme, G., Durán, V., Giardina, M., Novellino, P., De Santis, L., y Miguelissi, D. (2007). Exploraciones arqueológicas en laguna Llancanelo (Mendoza, Argentina). En F. Morello, M. Martinic, A. Prieto y G. Bahamonde (Eds.), Arqueología de Fuego-Patagonia. Levantando piedras, desenterrando huesos...y develando arcanos (pp. 453-466). Punta Arenas: Ediciones CEQUA.

Gil, A. F., Neme G. A. y Tykot, R. H. (2010). Isótopos estables y consumo de maíz en el centro occidente argentino: Tendencias temporales y espaciales. Chungará 42(2), 497-513.

(2011). Stable isotopes and human diet in central western Argentina. Journal of Archaeological Science 38(7), 1395-1404.

Gil, A., Neme, G., Ugan, A., y Tykot, R. (2012). Isótopos estables $\left({ }^{13} \mathrm{C},{ }^{15} \mathrm{~N},{ }^{18} \mathrm{O}\right)$ en la arqueología del sur de Mendoza. En A. Gil y G. Neme (Eds.), Paleobiogeografía en el Sur de Mendoza: Perspectivas Arqueológicas (pp. 135-156). Buenos Aires: Sociedad Argentina de Antropología.

Heaton, T. (1999). Spatial, species, and temporal variations in the ${ }^{13} \mathrm{C} /{ }^{12} \mathrm{C}$ ratios of $\mathrm{C}^{3}$ plants: Implications for paleodiets studies. Journal of Archaeological Science 26, 637-649.

Hedges, R. E., Clement, J. G., Thomas, C. D., y O'connell, T. C. (2007). Collagen turnover in the adult femoral mid-shaft: modeled from anthropogenic radiocarbon tracer measurements. American Journal of Physical Anthrology 133(2), 808-816.

Hedges, R., y Reynard, L. (2007). Nitrogen isotopes and the trophic level of humans in archaeology. Journal of Archaeological Science 34, 1240-1251.

Howland M. R., Corrs, L. T., Young, S. M., Jones, V., Jim, S., Van Der Merwe, N. J., Mitchell, A. D., y Evershed, R. P. (2003). Expression of the Dietary Isotope Signal in the Compound-specific $\delta^{13} \mathrm{C}$ Values of Pig Bone Lipids and Amino Acids. International Journal of Osteoarchaeology 13: 54-65

Isla, F. I., Ruiz Barket, E., Karquéz, J., y Urrutia, A. (2005) ENSO Signal Recognized by NDVI Images from Llancanelo Shallow Lake, Mendoza, Argentina. La Plata: XVI Congreso Geológico Argentino

Jim, S., Jones, V., Ambrose, S., y Evershed, R. (2006). Quantifying dietary macro-nutrient sources of carbon for bone collagen synthesis using natural abundance stable carbon isotope analysis. British Journal of Nutrition 95, 1055-1062.

Kellner, C., y Schoeninger, M. (2007). A simple carbon isotope model for reconstructing prehistoric human diet. American Journal of Physical Anthropology 133, 1112-1127.

Knudson, K. J. (2009). Oxygen isotope analysis in a land of environmental extremes: the complexities of isotopic work in the Andes. International Journal of Osteoarchaeology 19(2), 171-191.

Koch P, L. (1998). Isotopic reconstruction of past continental environments. Annual Review of Earth Planet Science 26, 573-613.

Longinelli, A. (1984). Oxygen isotopes in mammal bone phosphate: a new tool for paleohydrological and paleoclimatological research? Geochimica et Cosmochimica Acta 48, 385-390.

Longinelli, A., y Nuti, S. (1973). Oxygen isotope measurements of phosphate from fish teeth and bones. Earth and 
Planetary Science Letters 20(3), 337-340.

Luz, B., Kolodny, Y., y Horowitz, M., (1984). Fractionation of oxygen isotopes between mammalian bone-phosphate and environmental drinking water. Geochimica et Cosmochimica Acta 48(8), 1689-693.

Llano, C. (2012). Aprovechamiento de los recursos vegetales silvestres entre las sociedades cazadoras recolectoras del sur de Mendoza. (Tesis de doctorado inédita). Universidad Nacional de Comahue, Nuequén.

Llano, C., y Andreoni, D. (2012). Caracterización espacial y temporal en el uso de los recursos vegetales entre los grupos cazadores-recolectores del sur Mendocino durante el Holoceno. En G. Neme y A. Gil (Eds.), Paeoecología humana en el sur de Mendoza: perspectivas arqueológicas (pp. 57-84). Buenos Aires: Sociedad Argentina de Antropología.

Martínez Del Rio, C., Wolf, N., Carleton, S., y Gannes, Z. (2009). Isotopic ecology ten years after a call for more laboratory experiments. Biological Review 84, 91111.

Navarro, J. L., Barri, F. R., Maestri, D. M., Labuckas, D. o., y Martella, M. B. (2003). Physical characteristics and chemical composition of Lesser Rhea (Pterocnemia pennata) eggs from farmed populations. British Poultry Science 44, 586-590.

Neme, G., y Gil, A. (2008). Biogeografía humana en los andes meridionales: tendencias arqueológicas en el sur de Mendoza. Chungará, 40(1), 5-18.

(2012). El registro arqueológico del sur de Mendos en perspectiva biogeográfica. En G. Neme y A. Gil (Eds.), Paeoecología humana en el sur de Mendoza: perspectivas arqueológicas (pp. 255-280). Buenos Aires: Sociedad Argentina de Antropología.

Nicholas, G. P. (1998). Wetlands and Hunter-Gatherers: A Global Perspective. Current Anthropology 39(5), 720731.

Otaola, C., Giardina, M., Corbat, M., y Fernández, F. (2012). Zooarqueología en el sur de Mendoza: integrando perspectivas en un marco biogeográfico. En G. Neme y A. Gil (Eds.), Paeoecología humana en el sur de Mendoza: perspectivas arqueológicas (pp. 85-116). Buenos Aires: Sociedad Argentina de Antropología.

Panarello, H., Tessone A., y Zangrando, A. (2010). Isótopos estables en arqueología: principios teóricos, aspectos metodológicos y aplicaciones en Argentina. Xama 1923, 115-133.

Pate, D. (1997). Bone chemistry and paleodiet: reconstructing prehistoric subsistence settlement systems in Australia. Journal of Anthropological Archaeology 16, 130-
120.

Petracci, P. F., Ibáñez, H., Scorolli, A., Faillá, M., Blanco, D., Forcelli, D., Cozzani, N., De La Balze, V., Maclean, D., López Lanús, B., Carrizo, M., Sarria, R., Bogdaschewsky, R., Cereghetti, J., León, M., Díaz, L., Areco, A., Giovine, P., Bustamante, C., Veiga, J., Sotelo, M., Urioste, M., y Delarada, S. (2009a). Monitoreo poblacional de cauquenes migratorios (Chloephaga spp.) en las provincias de Buenos Aires y Río Negro, julio de 2008. Plan Nacional de Conservación y Manejo de Cauquenes. Buenos Aires, Argentina: Secretaría de Ambiente y Desarrollo Sustentable de la Nación, Jefatura de Gabinete de Ministros, Presidencia de la Nación.

Petracci, P., Cereghetti, J., Martín J., y Obed, S. (2009b). Dieta del Biguá (Phalacrocorax olivaceus) durante la primavera en el estuario de Bahía Blanca, Buenos Aires, Argentina. El Hornero 24(2), 73-78. Solamente esta citado 2009 agregar a y b o eliminar uno

Petzke, K. J., Benjamin, T., Fullerb, C., y Metges, C. (2010). Advances in natural stable isotope ratio analysis of human hair to determine nutritional and metabolic status. Current Opinion in Clinical Nutrition and Metabolic Care 13, 532-540

Phillips, D., y Gregg, J. (2003). Source partitioning using stable isotopes: coping with too many sources. Oecología 136, 261-269.

Phillips, D., y Koch, P. (2002). Incorporating concentration dependence in stable isotope mixing models. Oecologia 130, 114-1258

Phillips, D. L., Newsome, S. D., y Gregg, J. W. (2005). Combining sources in stable isotope mixing models: alternative methods. Oecologia 144, 520-527.

Sanhueza, L., y Falabella, F. (2010). Analysis of Stable Isotopes: From the Archaic to the Horticultural Communities in Central Chile. Current Anthropology 50(1), 127-136.

Schoeninger, M., y De Niro, M. (1984). Nitrogen and car- bon isotopic composition of bone collagen from marine and terrestrial animals. Gecochemica et Comochinica Acta 48, 625-639.

Schwarcz, H. P. (2000). Some biochemical aspects of carbon isotopic paleodiet studies. En S.H. Ambrose y M. A. Katzenberg (Eds.), Biochemical Approaches to Paleodietary Analysis (pp. 189-209). New York: Kluwer Academic/Plenum Publishers.

Sealy, J., Van Der Merwe, N., Lee-Thorp, J., y Lanham, J. (1987). Nitrogen isotopic ecology in southern Africa: Implications for environmental and dietary tracing. Gecochemica et Comochinica Acta 51, 2707-2717. 
Sponheimer, M. y Lee-Thorp, J. (1999). Oxygen isotopes in enamel carbonate and their ecological significance. Journal of Archaeological Science 26(6), 723-728.

Szpak, P., White, C. D., Longstaffe, F. J., Millaire, J. F., y Vásquez Sánchez, V. F. (2013). Carbon and Nitrogen Isotopic Survey of Northern Peruvian Plants: Baselines for Paleodietary and Paleoecological Studies. Plos One 8, 1-28.

Tessone, A. (2010). Arqueología y Ecología Isotópica. Estudio de isótopos estables de restos humanos del Holoceno tardio en Patagonia Meridional. (Tesis doctoral inédita). Facultad de Filosofía y Letras, Universidad de Buenos Aires, Buenos Aires.

Tessone, A., y Belardi, J. (2010). Evaluación del $\delta^{13} \mathrm{C}$ y $\delta^{15} \mathrm{~N}$ en el colágeno de herbívoros de las cuencas de los lagos Tar y San Martín (provincia de Santa Cruz, Patagonia). En M. De Nigris, P. M. Fernández, M. Giardina, A. F. Gil, M. A. Gutiérrez, A. Izeta, G. Neme y H. D. Yacobaccio (Eds.), Zooarqueología a principios del siglo XXI: aportes teóricos, metodológicos y casos de estudio (pp. 345-358). Buenos Aires: Ediciones Libros del Espinillo.

Tieszen, L., y Fagre, T. (1993). Carbon isotopic variability in modern and archaeological maize. Journal of Archaeological Science 20, 25-40.

Turner, B., Kamenov, G., Kingston, J. D., y Armelagos, G. J. (2009). Insights into immigration and social class at Machu Picchu, Peru based on oxygen, strontium, and lead isotopic analysis. Journal of Archaeological Science 36(2), 317-332.

Tykot, R. H. (2006). Isotope analyses and the histories of maize, En J. Staller, R. Tykot y B. Benz (Eds.), Histories of Maize (pp. 131-142). Massachusetts: Academic Press.

Tykot, R.H., Falabella, F., Planella, T., Aspillaga, E., Sanhueza, L., y Becker, C. (2009). Stable isotopes and archaeology in central Chile: methodological insights and interpretive problems for dietary reconstruction. International Journal of Osteoarchaeology 19, 156-170.

Turner, B., Kamenov, J. D., Kingston, J. D., y Armelagos, G. (2009). Insights into inmigration and social class at Machu Picchu, Perú, based on oxygen, strontium and lead analysis. Journal of Archaeological Science 36, 317-332.

Ugan, A., y Coltrain, J. (2011). Variation in collagen stable nitrogen values in black-tailed jackrabbits (Lepus californicus) in relation to small-scale differences in climate, soil, and topography. Journal of Archaeological Science 38(7), 1417-1429.

Ugan, A. y Coltrain, J. (2012) Stable isotopes, diet, and taphonomy: a look at using isotope-based dietary reconstructions to infer differential survivorship in zooarchaeological assemblages. Journal of Archaeological Science 39, 1401-1411.

Ugan, A., Neme, G., Gil, A., Coltrain, J., Tykot, R., y Novellino, P. (2012). Geographic variation in bone carbonate and water $\delta 180$ values in Mendoza, Argentina and their relationship to prehistoric economy and settlement. Journal of Archaeological Science 39(8), 2752-2763.

U.S. Department of Agriculture, A. R. S. (2009). USDA National Nutrient Database for Standard Reference, Release 22. Nutrient Data Laboratory Home Page. Recuperado de http://www.ars.usda.gov/ba/bhnrc/ ndl.

White, C. D., Spence, M. W., Stuart-Williams, H., y Schwarcz, H. P. (1998). Oxygen isotopes and the identification of geographical origins: the Valley of Oaxaca versus the Valley of Mexico. Journal of Archaeological Science 25(7), 643-655.

White, C. D., Spence, M.W., Longstaffe F. J., y Law, K. R. (2000). Testing the nature of Teotihuacán imperialism at Kaminaljuyú using phosphate oxygen-isotope ratios. Journal of Anthropological Research 56(4), 535-558.

White, C. D., Spence, M. W., Longstaffe, F. J., Stuart-Williams, H., y Law, K. R. (2002). Geographic identities of the sacrificial victims from the Feathered Serpent Pyramid, Teotihuacan: implications for the nature of state power. Latin American Antiquity 13(2), 217-236.

White, C. D., Longstaffe, F. J., y Law, K. R. (2004). Exploring the effects of environment, physiology and diet on oxygen isotope ratios in ancient Nubian bones and teeth. Journal of Archaeological Science 31(2), 233250. 
